\title{
Statistics for Poisson models of overlapping spheres
}

\author{
Daniel Hug, Günter Last, Zbyněk Pawlas and Wolfgang Weil
}

November 10, 2018

\begin{abstract}
The paper considers the stationary Poisson Boolean model with spherical grains and proposes a family of nonparametric estimators for the radius distribution. These estimators are based on observed distances and radii, weighted in an appropriate way. They are ratio-unbiased and asymptotically consistent for growing observation window. It is shown that the asymptotic variance exists and is given by a fairly explicit integral expression. Asymptotic normality is established under a suitable integrability assumption on the weight function. The paper also provides a short discussion of related estimators as well as a simulation study.
\end{abstract}

\section{Introduction}

We consider a stationary random closed set $Z$ in $\mathbb{R}^{d}(d \geq 2)$ which is given as a union of random balls of the form

$$
Z:=\bigcup_{n \geq 1} B\left(\xi_{n}, R_{n}\right)
$$

where $B(x, r)$ is a closed Euclidean ball with radius $r \geq 0$ centered at $x \in \mathbb{R}^{d}, \Phi:=\left\{\xi_{n}: n \geq 1\right\}$ is a stationary Poisson point process on $\mathbb{R}^{d}$, and the sequence $\left(R_{n}\right)_{n \geq 1}$ is independent of $\Phi$ and is formed by independent non-negative random variables with common distribution $\mathbb{G}$. Let $R$ be a generic random variable with distribution $\mathbb{G}$. We will always assume that it has a finite $2 d$-th moment, that is,

$$
\mathbb{E} R^{2 d}<\infty
$$

Definition (1.1) provides an important model in stochastic geometry with numerous applications in physics and materials science, for instance. The set $Z$ is called a stationary Boolean model with spherical grains. A simulated realization for $d=2$ is shown in Figure 1 .

It is a fundamental statistical problem to retrieve information on $\mathbb{G}$ based on an observation of $Z$ in a bounded window $W$. Our aim in this paper is to propose and study a family of nonparametric estimators of $\mathbb{G}$. The nonparametric estimation of the radius distribution $\mathbb{G}$ has been studied before; see [3, Chapter 5.6], [14] or [16]. In [16] a kernel estimator is obtained by the method of tangent points. The asymptotic properties of this estimator are studied in [8]. For earlier work on statistics for the Boolean model, we refer to [19, Chapter 3.3], [15] and the references therein.

In the following, we assume that all random elements are defined on an underlying probability space $(\Omega, \mathcal{F}, \mathbb{P})$. For a Borel set $A \subset \mathbb{R}^{d}$, we write $\Phi(A):=\operatorname{card}\left\{n \geq 1: \xi_{n} \in A\right\}$ and assume that $\Phi$ has a positive and finite intensity

$$
\gamma:=\mathbb{E} \Phi\left([0,1]^{d}\right)
$$

MSC 2010 subject classifications. Primary 60D05, 60G57, 52A21; secondary 60G55, 52A22, 52A20, 53C65, 46B20, $62 \mathrm{G} 05$.

Key words and phrases. Stochastic geometry, spatial statistics, contact distribution function, Boolean model, spherical typical grain, point process, nonparametric estimation, radius distribution, asymptotic normality. 
Throughout the paper, let $B$ be a compact convex set which contains the origin o and a non-degenerate segment. We call $B$ structuring element or gauge body, but we point out that $B$ need not be centrally symmetric or full-dimensional. The $B$-distance from a point $x \in \mathbb{R}^{d}$ to a set $A \subset \mathbb{R}^{d}$ is

$$
d_{B}(x, A):=\inf \{r \geq 0:(x+r B) \cap A \neq \emptyset\} \in[0, \infty] .
$$

Clearly, if o $\in \operatorname{int} B$ and $A$ is nonempty and closed, then the infimum is a minimum. The most common structuring element is the unit ball $B(\mathrm{o}, 1)$, for which we also write $B^{d}$ and which is based on a scalar product and a norm denoted by $\|\cdot\|$. For given $x \notin Z$, almost surely $d_{B}(x, Z)<\infty$ whenever $R$ satisfies $\mathbb{P}(R>0)>0$. We always assume that this condition is fulfilled. Then almost surely there is a unique $n \in \mathbb{N}$ (that is, a ball $B\left(\xi_{n}, R_{n}\right)$ ) such that $\left(x+d_{B}(x, Z) B\right) \cap B\left(\xi_{n}, R_{n}\right) \neq \emptyset$ (see [10. Lemma 3.1] or [20, Lemma 9.5.1]). In this case, we define $r_{B}(x, Z)$ as $R_{n}$. Figure 1 illustrates the definition of $d_{B}(x, Z)$ and $r_{B}(x, Z)$ for $x \notin Z$ and $B=B^{2}$.

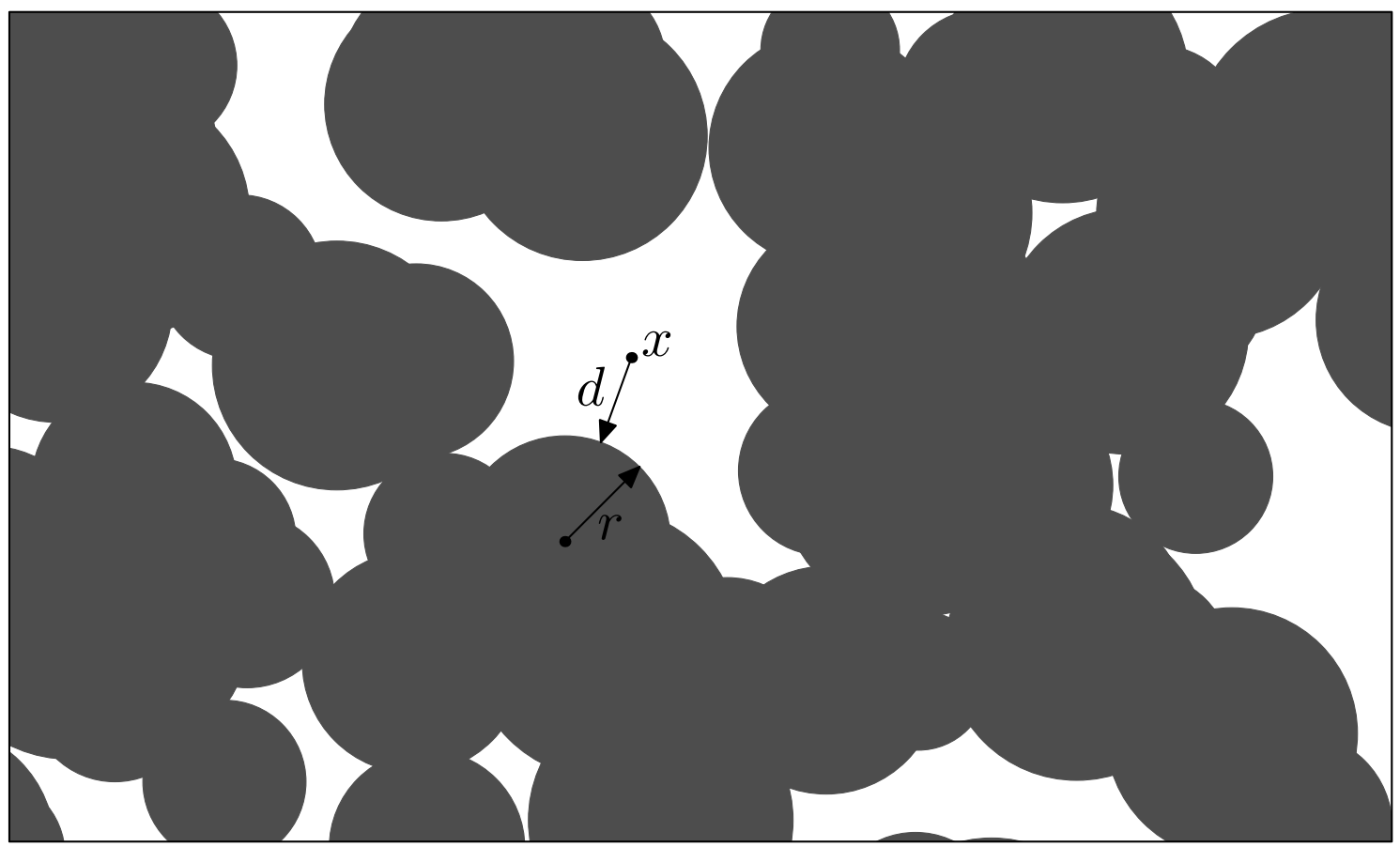

Figure 1: A simulated realization of a planar stationary Boolean model $Z$ with spherical grains observed in a rectangular observation window. The symbol $d$ denotes $d_{B^{2}}(x, Z)$ and $r$ stands for $r_{B^{2}}(x, Z)$.

For $s, r \geq 0$, we write $B_{s, r}:=s B \oplus r B^{d}$ for the Minkowski sum of $s B$ and $r B^{d}$. Let $|A|_{d}$ denote the $d$-dimensional Lebesgue measure of a set $A \subset \mathbb{R}^{d}$, let $\kappa_{k}:=\left|B^{k}\right|_{k}=\pi^{k / 2} / \Gamma(1+k / 2)$ denote the volume of the $k$-dimensional unit ball and write $V_{j}(B)$ for the $j$-th intrinsic volume of $B$ (see [20, Chapter 14.3]). Then, for $t \in \mathbb{R}^{+}:=[0, \infty)$, the empty space function $F_{B}$ of $Z$ is given by

$$
\begin{aligned}
F_{B}(t) & :=\mathbb{P}\left(d_{B}(\mathrm{o}, Z) \leq t\right)=\mathbb{P}(Z \cap t B \neq \emptyset)=1-\exp \left\{-\gamma \mathbb{E}\left|B_{t, R}\right|_{d}\right\} \\
& =1-\exp \left\{-\gamma \sum_{j=0}^{d} \kappa_{d-j} V_{j}(B) t^{j} \mathbb{E} R^{d-j}\right\}
\end{aligned}
$$

The empty space function is a useful summary statistics of random sets (see [19, 5]). In the case of a strictly convex gauge body $B$ a detailed study of $F_{B}$ for (non-stationary) germ-grain models can be found in [9]. We denote the complementary empty space function by $\bar{F}_{B}(t):=1-F_{B}(t)$. As a 
consequence of [10, Theorem 3.2], we get for all measurable functions $\tilde{g}:[0, \infty] \times \mathbb{R}^{+} \rightarrow \mathbb{R}^{+}$such that $\tilde{g}(0, r)=\tilde{g}(\infty, r)=0, r \in \mathbb{R}^{+}$, and all $x \in \mathbb{R}^{d}$ that

$$
\mathbb{E} \tilde{g}\left(d_{B}(x, Z), r_{B}(x, Z)\right)=\gamma \int_{0}^{\infty} \int_{0}^{\infty} \tilde{g}(t, r) h_{B}(t, r) \bar{F}_{B}(t) \mathrm{d} t \mathbb{G}(\mathrm{d} r)
$$

with

$$
h_{B}(t, r):=\sum_{j=0}^{d-1}(j+1) \kappa_{d-1-j} V_{j+1}(B) r^{d-1-j} t^{j},
$$

for $t, r \in[0, \infty)$; see also [20, Theorem 9.5.2]. Note that on the left-hand side of (1.4) the restriction to $\left\{0<d_{B}(x, Z)<\infty\right\}$ is expressed by the condition $\tilde{g}(0, r)=\tilde{g}(\infty, r)=0$.

For Borel sets $C \subset \mathbb{R}^{+}, A \subset \mathbb{R}^{d}$ and a measurable function $f:[0, \infty] \rightarrow \mathbb{R}^{+}$with $f(0)=f(\infty)=$ 0 , we define a random measure $\eta_{A}$ by

$$
\eta_{A}(C):=\int_{A} \mathbf{1}\left\{r_{B}(x, Z) \in C\right\} f\left(d_{B}(x, Z)\right) h_{B}\left(d_{B}(x, Z), r_{B}(x, Z)\right)^{-1} \mathrm{~d} x,
$$

where $1\{\cdot\}$ denotes the indicator function. Here we put $0 / 0:=0$. Thus, in particular, the integration effectively extends over the complement $Z^{c}:=\left\{x \in \mathbb{R}^{d}: d_{B}(x, Z)>0\right\}$ of $Z$. Throughout the paper we shall assume that

$$
0<\beta:=\int_{0}^{\infty} f(t) \bar{F}_{B}(t) \mathrm{d} t<\infty .
$$

In view of (1.3) this is a rather weak assumption on $f$. Moreover, we assume that the origin is an interior point of $B$ if $\mathbb{P}(R=0)>0$. This assumption ensures that $h_{B}(t, r)>0$ for $t \in(0, \infty)$ and $\mathbb{G}$-almost all $r \in \mathbb{R}^{+}$. By Fubini's theorem and (1.4), we obtain

$$
\mathbb{E} \eta_{A}(C)=\gamma \beta|A|_{d} \mathbb{G}(C) .
$$

Consider a compact convex observation window $W \subset \mathbb{R}^{d}$ with $|W|_{d}>0$. We propose an estimator $\widehat{\mathbb{G}}$ for $\mathbb{G}$ based on the information contained in the data $\left\{\left(d_{B}(x, Z), r_{B}(x, Z)\right): x \in W \backslash Z\right\}$. Note that these data may also require information from outside $W$. The estimator is given by

$$
\widehat{\mathbb{G}}(C):=\frac{\eta_{W}(C)}{\eta_{W}\left(\mathbb{R}^{+}\right)},
$$

where $C \subset \mathbb{R}^{+}$is a Borel set. If the denominator in $(1.8)$ is zero, then the numerator is zero as well, and we use the convention $0 / 0:=0$. From (1.7) we see that $\mathbb{E} \eta_{W}(C)=\gamma \beta|W|_{d} \mathbb{G}(C)$ and $\mathbb{E} \eta_{W}\left(\mathbb{R}^{+}\right)=$ $\gamma \beta|W|_{d}$. This means that $\widehat{\mathbb{G}}$ is a ratio-unbiased estimator of $\mathbb{G}$.

The paper is organized as follows. In Section 2 we study second order properties of (1.5). We show that the asymptotic variance exists and is given by a fairly explicit integral expression. Consequently, the estimator (1.8) is asymptotically weakly consistent as the compact convex observation window $W$ is expanding. Strong consistency follows from the spatial ergodic theorem. Section 3 contains the proof of asymptotic normality under an integrability assumption on the function $f$. In Section 4 we consider the estimator $\widehat{\mathbb{G}}$ in the plane and for the spherical $(B=B(\mathrm{o}, 1))$ as well as for the linear case ( $B$ a segment). We also discuss some related estimators. A simulation study is performed to compare the behaviour of different discrete versions of these estimators of the radius distribution $\mathbb{G}$. 


\section{Second order properties}

For a Borel set $A \subset \mathbb{R}^{d}$, we define the restricted Boolean model as

$$
Z(A):=\bigcup_{n: \xi_{n} \in A} B\left(\xi_{n}, R_{n}\right)
$$

Clearly, $Z(A)$ is not stationary unless $A=\mathbb{R}^{d}$. Further, for $t \in \mathbb{R}^{+}$the complementary empty space function of $Z(A)$ with respect to $x \in \mathbb{R}^{d}$ is defined by

$$
\begin{aligned}
\bar{F}_{B}^{A}(x ; t) & :=\mathbb{P}\left(d_{B}(x, Z(A))>t\right)=\mathbb{P}((x+t B) \cap Z(A)=\emptyset) \\
& =\mathbb{E} \prod_{n \geq 1}\left(1-\mathbf{1}\left\{(x+t B) \cap B\left(\xi_{n}, R_{n}\right) \neq \emptyset\right\} \mathbf{1}\left\{\xi_{n} \in A\right\}\right) \\
& =\exp \left\{-\gamma \mathbb{E} \int_{\mathbb{R}^{d}} \mathbf{1}\{(x+t B) \cap B(y, R) \neq \emptyset\} \mathbf{1}\{y \in A\} \mathrm{d} y\right\} \\
& =\exp \left\{-\gamma \mathbb{E}\left|\left(x+B_{t, R}\right) \cap A\right|_{d}\right\} .
\end{aligned}
$$

In particular, we have $\bar{F}_{B}^{\mathbb{R}^{d}}(x ; t)=\bar{F}_{B}(t)$.

For Borel sets $A_{1}, A_{2} \subset \mathbb{R}^{d}$ and $t_{1}, t_{2} \in \mathbb{R}^{+}$, it will be convenient to introduce the complementary second-order empty space function with respect to $x_{1}, x_{2} \in \mathbb{R}^{d}$ as

$$
\begin{aligned}
\bar{F}_{B}^{A_{1}, A_{2}}\left(x_{1}, x_{2} ; t_{1}, t_{2}\right):=\mathbb{P}\left(d_{B}\left(x_{1}, Z\left(A_{1}\right)\right)>t_{1}, d_{B}\left(x_{2}, Z\left(A_{2}\right)\right)>t_{2}\right) \\
=\mathbb{P}\left(\left(x_{1}+t_{1} B\right) \cap Z\left(A_{1}\right)=\emptyset,\left(x_{2}+t_{2} B\right) \cap Z\left(A_{2}\right)=\emptyset\right) \\
=\mathbb{E} \prod_{n \geq 1}\left(1-\mathbf{1}\left\{\left(x_{1}+t_{1} B\right) \cap B\left(\xi_{n}, R_{n}\right) \neq \emptyset\right\} \mathbf{1}\left\{\xi_{n} \in A_{1}\right\}\right) \\
\quad \times\left(1-\mathbf{1}\left\{\left(x_{2}+t_{2} B\right) \cap B\left(\xi_{n}, R_{n}\right) \neq \emptyset\right\} \mathbf{1}\left\{\xi_{n} \in A_{2}\right\}\right) \\
=\exp \left\{-\gamma \mathbb{E}\left|\left(\left(x_{1}+B_{t_{1}, R}\right) \cap A_{1}\right) \cup\left(\left(x_{2}+B_{t_{2}, R}\right) \cap A_{2}\right)\right|_{d}\right\} .
\end{aligned}
$$

This function is related to the second-order contact distribution function which is studied in [1].

In order to obtain a more concise statement in the subsequent Lemma 2.1 (and again in the proof of Theorem 3.1), we introduce for given Borel sets $A_{1}, A_{2} \subset \mathbb{R}^{d}$ two functions, $I_{1}\left(A_{1}, A_{2}\right)$ and $I_{2}\left(A_{1}, A_{2}\right)$, depending on the arguments $\left(x_{1}, x_{2}, y, r\right) \in\left(\mathbb{R}^{d}\right)^{3} \times \mathbb{R}^{+}$and $\left(x_{1}, x_{2}, y_{1}, y_{2}, r_{1}, r_{2}\right) \in\left(\mathbb{R}^{d}\right)^{4} \times\left(\mathbb{R}^{+}\right)^{2}$, respectively, which are defined by

$$
I_{1}\left(A_{1}, A_{2}\right)\left(x_{1}, x_{2}, y, r\right):=\mathbf{1}\left\{y \in A_{1} \cap A_{2}\right\} \bar{F}_{B}^{A_{1}, A_{2}}\left(x_{1}, x_{2} ; d_{B}\left(x_{1}, B(y, r)\right), d_{B}\left(x_{2}, B(y, r)\right)\right)
$$

and

$$
\begin{aligned}
& I_{2}\left(A_{1}, A_{2}\right)\left(x_{1}, x_{2}, y_{1}, y_{2}, r_{1}, r_{2}\right):=\mathbf{1}\left\{y_{1} \in A_{1}\right\} \mathbf{1}\left\{y_{2} \in A_{2}\right\} \\
& \times\left[\left(1-\mathbf{1}\left\{y_{2} \in A_{1}\right\} \mathbf{1}\left\{d_{B}\left(x_{1}, B\left(y_{2}, r_{2}\right)\right) \leq d_{B}\left(x_{1}, B\left(y_{1}, r_{1}\right)\right)\right\}\right)\right. \\
& \quad \times\left(1-\mathbf{1}\left\{y_{1} \in A_{2}\right\} \mathbf{1}\left\{d_{B}\left(x_{2}, B\left(y_{1}, r_{1}\right)\right) \leq d_{B}\left(x_{2}, B\left(y_{2}, r_{2}\right)\right)\right\}\right) \\
& \quad \times \bar{F}_{B}^{A_{1}, A_{2}}\left(x_{1}, x_{2} ; d_{B}\left(x_{1}, B\left(y_{1}, r_{1}\right)\right), d_{B}\left(x_{2}, B\left(y_{2}, r_{2}\right)\right)\right) \\
& \left.\quad-\bar{F}_{B}^{A_{1}}\left(x_{1} ; d_{B}\left(x_{1}, B\left(y_{1}, r_{1}\right)\right)\right) \bar{F}_{B}^{A_{2}}\left(x_{2} ; d_{B}\left(x_{2}, B\left(y_{2}, r_{2}\right)\right)\right)\right] .
\end{aligned}
$$

If the arguments of these two functions are clear from the context, they are sometimes omitted. 
Lemma 2.1. Let $A_{1}, A_{2} \subset \mathbb{R}^{d}$ be Borel sets and let $x_{1}, x_{2} \in \mathbb{R}^{d}$. If $\tilde{g}:[0, \infty] \times \mathbb{R}^{+} \rightarrow \mathbb{R}^{+}$is a measurable function with $\tilde{g}(0, r)=\tilde{g}(\infty, r)=0$ for $r \in \mathbb{R}^{+}$, then

$$
\begin{aligned}
\operatorname{Cov}\left(\tilde{g}\left(d_{B}\left(x_{1}, Z\left(A_{1}\right)\right), r_{B}\left(x_{1}, Z\left(A_{1}\right)\right)\right), \tilde{g}\left(d_{B}\left(x_{2}, Z\left(A_{2}\right)\right), r_{B}\left(x_{2}, Z\left(A_{2}\right)\right)\right)\right) \\
=\gamma \int_{0}^{\infty} \int_{\mathbb{R}^{d}} \tilde{g}\left(d_{B}\left(x_{1}, B(y, r)\right), r\right) \tilde{g}\left(d_{B}\left(x_{2}, B(y, r)\right), r\right) I_{1}\left(A_{1}, A_{2}\right)\left(x_{1}, x_{2}, y, r\right) \mathrm{d} y \mathbb{G}(\mathrm{d} r) \\
+\gamma^{2} \int_{0}^{\infty} \int_{0}^{\infty} \int_{\mathbb{R}^{d}} \int_{\mathbb{R}^{d}} \tilde{g}\left(d_{B}\left(x_{1}, B\left(y_{1}, r_{1}\right)\right), r_{1}\right) \tilde{g}\left(d_{B}\left(x_{2}, B\left(y_{2}, r_{2}\right)\right), r_{2}\right) \\
\quad \times I_{2}\left(A_{1}, A_{2}\right)\left(x_{1}, x_{2}, y_{1}, y_{2}, r_{1}, r_{2}\right) \mathrm{d} y_{1} \mathrm{~d} y_{2} \mathbb{G}\left(\mathrm{d} r_{1}\right) \mathbb{G}\left(\mathrm{d} r_{2}\right) .
\end{aligned}
$$

Proof. For $n \in \mathbb{N}, x \in \mathbb{R}^{d}$, and $i \in\{1,2\}$, we define the event

$$
D_{n}^{(i)}(x):=\left\{d_{B}\left(x, \bigcup_{k \neq n: \xi_{k} \in A_{i}} B\left(\xi_{k}, R_{k}\right)\right)>d_{B}\left(x, B\left(\xi_{n}, R_{n}\right)\right)\right\} .
$$

Then

$$
\begin{aligned}
\mathbb{E} \tilde{g} & \left(d_{B}\left(x_{1}, Z\left(A_{1}\right)\right), r_{B}\left(x_{1}, Z\left(A_{1}\right)\right)\right) \tilde{g}\left(d_{B}\left(x_{2}, Z\left(A_{2}\right)\right), r_{B}\left(x_{2}, Z\left(A_{2}\right)\right)\right) \\
= & \mathbb{E} \sum_{n: \xi_{n} \in A_{1}} \sum_{m: \xi_{m} \in A_{2}} \mathbf{1}_{D_{n}^{(1)}\left(x_{1}\right) \cap D_{m}^{(2)}\left(x_{2}\right)} \tilde{g}\left(d_{B}\left(x_{1}, B\left(\xi_{n}, R_{n}\right)\right), R_{n}\right) \tilde{g}\left(d_{B}\left(x_{2}, B\left(\xi_{m}, R_{m}\right)\right), R_{m}\right) \\
= & \mathbb{E} \sum_{n: \xi_{n} \in A_{1} \cap A_{2}} \mathbf{1}_{D_{n}^{(1)}\left(x_{1}\right) \cap D_{n}^{(2)}\left(x_{2}\right)} \tilde{g}\left(d_{B}\left(x_{1}, B\left(\xi_{n}, R_{n}\right)\right), R_{n}\right) \tilde{g}\left(d_{B}\left(x_{2}, B\left(\xi_{n}, R_{n}\right)\right), R_{n}\right) \\
& +\mathbb{E} \sum_{n \neq m: \xi_{n} \in A_{1}, \xi_{m} \in A_{2}} \sum_{D_{n}^{(1)}\left(x_{1}\right) \cap D_{m}^{(2)}\left(x_{2}\right)} \tilde{g}\left(d_{B}\left(x_{1}, B\left(\xi_{n}, R_{n}\right)\right), R_{n}\right) \tilde{g}\left(d_{B}\left(x_{2}, B\left(\xi_{m}, R_{m}\right)\right), R_{m}\right) \\
= & : J_{1}+J_{2} .
\end{aligned}
$$

Applying Mecke's formula (see [20, Corollary 3.2.3]), we obtain

$$
\begin{aligned}
J_{1}=\gamma & \int_{0}^{\infty} \int_{A_{1} \cap A_{2}} \tilde{g}\left(d_{B}\left(x_{1}, B(y, r)\right), r\right) \tilde{g}\left(d_{B}\left(x_{2}, B(y, r)\right), r\right) \\
& \times \mathbb{P}\left(d_{B}\left(x_{1}, Z\left(A_{1}\right)\right)>d_{B}\left(x_{1}, B(y, r)\right), d_{B}\left(x_{2}, Z\left(A_{2}\right)\right)>d_{B}\left(x_{2}, B(y, r)\right)\right) \mathrm{d} y \mathbb{G}(\mathrm{d} r)
\end{aligned}
$$

and

$$
\begin{aligned}
J_{2}=\gamma^{2} & \int_{0}^{\infty} \int_{0}^{\infty} \int_{A_{2}} \int_{A_{1}} \tilde{g}\left(d_{B}\left(x_{1}, B\left(y_{1}, r_{1}\right)\right), r_{1}\right) \tilde{g}\left(d_{B}\left(x_{2}, B\left(y_{2}, r_{2}\right)\right), r_{2}\right) \\
& \times \mathbb{E} \mathbf{1}\left\{d_{B}\left(x_{1}, Z_{y_{2}}\left(A_{1}\right)\right)>d_{B}\left(x_{1}, B\left(y_{1}, r_{1}\right)\right)\right\} \mathbf{1}\left\{d_{B}\left(x_{2}, Z_{y_{1}}\left(A_{2}\right)\right)>d_{B}\left(x_{2}, B\left(y_{2}, r_{2}\right)\right)\right\} \\
& \times \mathrm{d} y_{1} \mathrm{~d} y_{2} \mathbb{G}\left(\mathrm{d} r_{1}\right) \mathbb{G}\left(\mathrm{d} r_{2}\right),
\end{aligned}
$$

where $Z_{y_{2}}\left(A_{1}\right)=Z\left(A_{1}\right) \cup B\left(y_{2}, r_{2}\right)$ if $y_{2} \in A_{1}$ and $Z_{y_{2}}\left(A_{1}\right)=Z\left(A_{1}\right)$ if $y_{2} \notin A_{1}$. Analogously, 
$Z_{y_{1}}\left(A_{2}\right)=Z\left(A_{2}\right) \cup B\left(y_{1}, r_{1}\right)$ if $y_{1} \in A_{2}$ and $Z_{y_{1}}\left(A_{2}\right)=Z\left(A_{2}\right)$ if $y_{1} \notin A_{2}$. Hence,

$$
\begin{aligned}
J_{2}=\gamma^{2} & \int_{0}^{\infty} \int_{0}^{\infty} \int_{A_{2}} \int_{A_{1}} \tilde{g}\left(d_{B}\left(x_{1}, B\left(y_{1}, r_{1}\right)\right), r_{1}\right) \tilde{g}\left(d_{B}\left(x_{2}, B\left(y_{2}, r_{2}\right)\right), r_{2}\right) \\
& \times\left(1-\mathbf{1}\left\{y_{2} \in A_{1}\right\} \mathbf{1}\left\{d_{B}\left(x_{1}, B\left(y_{2}, r_{2}\right)\right) \leq d_{B}\left(x_{1}, B\left(y_{1}, r_{1}\right)\right)\right\}\right) \\
& \times\left(1-\mathbf{1}\left\{y_{1} \in A_{2}\right\} \mathbf{1}\left\{d_{B}\left(x_{2}, B\left(y_{1}, r_{1}\right)\right) \leq d_{B}\left(x_{2}, B\left(y_{2}, r_{2}\right)\right)\right\}\right) \\
& \times \mathbb{P}\left(d_{B}\left(x_{1}, Z\left(A_{1}\right)\right)>d_{B}\left(x_{1}, B\left(y_{1}, r_{1}\right)\right), d_{B}\left(x_{2}, Z\left(A_{2}\right)\right)>d_{B}\left(x_{2}, B\left(y_{2}, r_{2}\right)\right)\right) \\
& \times \mathrm{d} y_{1} \mathrm{~d} y_{2} \mathbb{G}\left(\mathrm{d} r_{1}\right) \mathbb{G}\left(\mathrm{d} r_{2}\right) .
\end{aligned}
$$

Finally,

$$
\begin{array}{r}
\mathbb{E} \tilde{g}\left(d_{B}\left(x_{1}, Z\left(A_{1}\right)\right), r_{B}\left(x_{1}, Z\left(A_{1}\right)\right)\right)=\mathbb{E} \sum_{n: \xi_{n} \in A_{1}} \mathbf{1}_{D_{n}^{(1)}\left(x_{1}\right)} \tilde{g}\left(d_{B}\left(x_{1}, B\left(\xi_{n}, R_{n}\right)\right), R_{n}\right) \\
=\gamma \int_{0}^{\infty} \int_{A_{1}} \tilde{g}\left(d_{B}\left(x_{1}, B\left(y_{1}, r_{1}\right)\right), r_{1}\right) \bar{F}_{B}^{A_{1}}\left(x_{1} ; d_{B}\left(x_{1}, B\left(y_{1}, r_{1}\right)\right)\right) \mathrm{d} y_{1} \mathbb{G}\left(\mathrm{d} r_{1}\right) .
\end{array}
$$

Our aim is to analyze the second-order properties of the random measure $\eta_{A}$ given by (1.5). For this reason, we work with the complementary second-order empty space function (2.2). For $A_{1}=A_{2}=\mathbb{R}^{d}$, $t_{1}, t_{2} \in \mathbb{R}^{+}$, and $u=x_{2}-x_{1}$, by the stationarity of $Z$ this function turns into

$$
\begin{aligned}
\bar{F}_{B}^{(2)}\left(u ; t_{1}, t_{2}\right) & :=\mathbb{P}\left(d_{B}(\mathrm{o}, Z)>t_{1}, d_{B}(u, Z)>t_{2}\right) \\
& =\exp \left\{-\gamma \mathbb{E}\left|B_{t_{1}, R} \cup\left(u+B_{t_{2}, R}\right)\right|_{d}\right\} \\
& =\bar{F}_{B}\left(t_{1}\right) \bar{F}_{B}\left(t_{2}\right) \exp \left\{\gamma \mathbb{E} \kappa_{B}\left(u ; t_{1}, t_{2}, R\right)\right\},
\end{aligned}
$$

where

$$
\kappa_{B}\left(u ; t_{1}, t_{2}, r\right):=\left|B_{t_{1}, r} \cap\left(u+B_{t_{2}, r}\right)\right|_{d} .
$$

Observe that for any $u \in \mathbb{R}^{d}$ and $t_{1}, t_{2} \in \mathbb{R}^{+}$, we have

$$
\bar{F}_{B}^{(2)}\left(u ; t_{1}, t_{2}\right) \geq \bar{F}_{B}\left(t_{1}\right) \bar{F}_{B}\left(t_{2}\right)
$$

and

$$
\bar{F}_{B}^{(2)}\left(u ; t_{1}, t_{2}\right) \leq \exp \left\{-\frac{\gamma}{2} \mathbb{E}\left(\left|B_{t_{1}, R}\right|_{d}+\left|B_{t_{2}, R}\right|_{d}\right)\right\}=\sqrt{\bar{F}_{B}\left(t_{1}\right) \bar{F}_{B}\left(t_{2}\right)} .
$$

These inequalities will be used subsequently. In addition, we shall need the assumption

$$
\int_{0}^{\infty} f(t) \mathrm{e}^{-c t} \mathrm{~d} t<\infty
$$

where $c:=4^{-1} \gamma \kappa_{d-1} V_{1}(B) \mathbb{E} R^{d-1}<\infty$ and $c>0$ since $V_{1}(B)>0$ (recall that $B$ contains a nondegenerate line segment) and $\mathbb{P}(R>0)>0$. 
Proposition 2.2. Assume that (2.8) is satisfied. If $C \subset \mathbb{R}^{+}$is a Borel set and $W_{1}, W_{2} \subset \mathbb{R}^{d}$ are compact convex sets, then

$$
\operatorname{Cov}\left(\eta_{W_{1}}(C), \eta_{W_{2}}(C)\right)=\int_{\mathbb{R}^{d}}\left|W_{1} \cap\left(W_{2}-u\right)\right|_{d}\left[\gamma \tau_{1}(C, u)+\gamma^{2} \tau_{2}(C, u)\right] \mathrm{d} u,
$$

where

$$
\begin{aligned}
\tau_{1}(C, u):=\int_{C} \int_{\mathbb{R}^{d}} \frac{f\left(d_{B}(x, B(\mathrm{o}, r))\right)}{h_{B}\left(d_{B}(x, B(\mathrm{o}, r)), r\right)} \frac{f\left(d_{B}(u+x, B(\mathrm{o}, r))\right)}{h_{B}\left(d_{B}(u+x, B(\mathrm{o}, r)), r\right)} \\
\quad \times \bar{F}_{B}^{(2)}\left(u ; d_{B}(x, B(\mathrm{o}, r)), d_{B}(u+x, B(\mathrm{o}, r))\right) \mathrm{d} x \mathbb{G}(\mathrm{d} r)
\end{aligned}
$$

and

$$
\begin{aligned}
\tau_{2}(C, u):=\int_{C} \int_{C} \int_{\mathbb{R}^{d}} & \int_{\mathbb{R}^{d}} \frac{f\left(d_{B}\left(x_{1}, B\left(\mathrm{o}, r_{1}\right)\right)\right)}{h_{B}\left(d_{B}\left(x_{1}, B\left(\mathrm{o}, r_{1}\right)\right), r_{1}\right)} \frac{f\left(d_{B}\left(x_{2}, B\left(\mathrm{o}, r_{2}\right)\right)\right)}{h_{B}\left(d_{B}\left(x_{2}, B\left(\mathrm{o}, r_{2}\right)\right), r_{2}\right)} \\
& \times q\left(u ; x_{1}, x_{2}, r_{1}, r_{2}\right) \mathrm{d} x_{1} \mathrm{~d} x_{2} \mathbb{G}\left(\mathrm{d} r_{1}\right) \mathbb{G}\left(\mathrm{d} r_{2}\right),
\end{aligned}
$$

for $u \in \mathbb{R}^{d}$, and

$$
\begin{aligned}
& q\left(u ; x_{1}, x_{2}, r_{1}, r_{2}\right) \\
& :=\mathbf{1}\left\{d_{B}\left(x_{2}, B\left(u, r_{2}\right)\right)>d_{B}\left(x_{1}, B\left(\mathrm{o}, r_{1}\right)\right)\right\} \mathbf{1}\left\{d_{B}\left(x_{1}, B\left(-u, r_{1}\right)\right)>d_{B}\left(x_{2}, B\left(\mathrm{o}, r_{2}\right)\right)\right\} \\
& \quad \times \bar{F}_{B}^{(2)}\left(u ; d_{B}\left(x_{1}, B\left(\mathrm{o}, r_{1}\right)\right), d_{B}\left(x_{2}, B\left(\mathrm{o}, r_{2}\right)\right)\right)-\bar{F}_{B}\left(d_{B}\left(x_{1}, B\left(\mathrm{o}, r_{1}\right)\right)\right) \bar{F}_{B}\left(d_{B}\left(x_{2}, B\left(\mathrm{o}, r_{2}\right)\right)\right),
\end{aligned}
$$

for $x_{1}, x_{2} \in \mathbb{R}^{d}$ and $r_{1}, r_{2} \in \mathbb{R}^{+}$.

Proof. To abbreviate the notation, we define the function

$$
g(t, r):=\mathbf{1}\{r \in C\} f(t) h_{B}(t, r)^{-1},
$$

for $t \in[0, \infty]$ and $r \in \mathbb{R}^{+}$, with the previous conventions in the cases where $t \in\{0, \infty\}$. Recall also that $h_{B}(t, r)>0$ for $t \in(0, \infty)$ and $\mathbb{G}$-almost all $r \in \mathbb{R}^{+}$. Using Fubini's theorem and stationarity, we get

$$
\begin{aligned}
& \operatorname{Cov}\left(\eta_{W_{1}}(C), \eta_{W_{2}}(C)\right) \\
& \quad=\int_{W_{1}} \int_{W_{2}} \operatorname{Cov}\left(g\left(d_{B}\left(x_{1}, Z\right), r_{B}\left(x_{1}, Z\right)\right), g\left(d_{B}\left(x_{2}, Z\right), r_{B}\left(x_{2}, Z\right)\right)\right) \mathrm{d} x_{2} \mathrm{~d} x_{1} \\
& \quad=\int_{\mathbb{R}^{d}}\left|W_{1} \cap\left(W_{2}-u\right)\right|_{d} \operatorname{Cov}\left(g\left(d_{B}(\mathrm{o}, Z), r_{B}(\mathrm{o}, Z)\right), g\left(d_{B}(u, Z), r_{B}(u, Z)\right)\right) \mathrm{d} u
\end{aligned}
$$

By Lemma2.1 with $A_{1}=A_{2}=\mathbb{R}^{d}, x_{1}=\mathrm{o}$ and $x_{2}=u$, we obtain that

$$
\operatorname{Cov}\left(g\left(d_{B}(\mathrm{o}, Z), r_{B}(\mathrm{o}, Z)\right), g\left(d_{B}(u, Z), r_{B}(u, Z)\right)\right)=J_{1}(u)+J_{21}(u)-J_{22},
$$

where

$$
\begin{aligned}
J_{1}(u):=\gamma \int_{0}^{\infty} & \int_{\mathbb{R}^{d}} g\left(d_{B}(\mathrm{o}, B(x, r)), r\right) g\left(d_{B}(u, B(x, r)), r\right) \\
& \times \bar{F}_{B}^{(2)}\left(u ; d_{B}(\mathrm{o}, B(x, r)), d_{B}(u, B(x, r))\right) \mathrm{d} x \mathbb{G}(\mathrm{d} r),
\end{aligned}
$$




$$
\begin{aligned}
J_{21}(u):=\gamma^{2} & \int_{0}^{\infty} \int_{0}^{\infty} \int_{\mathbb{R}^{d}} \int_{\mathbb{R}^{d}} g\left(d_{B}\left(\mathrm{o}, B\left(x_{1}, r_{1}\right)\right), r_{1}\right) g\left(d_{B}\left(\mathrm{o}, B\left(x_{2}, r_{2}\right)\right), r_{2}\right) \\
& \times \mathbf{1}\left\{d_{B}\left(-u, B\left(x_{2}, r_{2}\right)\right)>d_{B}\left(\mathrm{o}, B\left(x_{1}, r_{1}\right)\right)\right\} \\
& \times \mathbf{1}\left\{d_{B}\left(u, B\left(x_{1}, r_{1}\right)\right)>d_{B}\left(\mathrm{o}, B\left(x_{2}, r_{2}\right)\right)\right\} \\
& \times \bar{F}_{B}^{(2)}\left(u ; d_{B}\left(\mathrm{o}, B\left(x_{1}, r_{1}\right)\right), d_{B}\left(\mathrm{o}, B\left(x_{2}, r_{2}\right)\right)\right) \mathrm{d} x_{1} \mathrm{~d} x_{2} \mathbb{G}\left(\mathrm{d} r_{1}\right) \mathbb{G}\left(\mathrm{d} r_{2}\right),
\end{aligned}
$$

and

$$
\begin{aligned}
J_{22}:=\gamma^{2} & \int_{0}^{\infty} \int_{0}^{\infty} \int_{\mathbb{R}^{d}} \int_{\mathbb{R}^{d}} g\left(d_{B}\left(\mathrm{o}, B\left(x_{1}, r_{1}\right)\right), r_{1}\right) g\left(d_{B}\left(\mathrm{o}, B\left(x_{2}, r_{2}\right)\right), r_{2}\right) \\
& \times \bar{F}_{B}\left(d_{B}\left(\mathrm{o}, B\left(x_{1}, r_{1}\right)\right)\right) \bar{F}_{B}\left(d_{B}\left(\mathrm{o}, B\left(x_{2}, r_{2}\right)\right)\right) \mathrm{d} x_{1} \mathrm{~d} x_{2} \mathbb{G}\left(\mathrm{d} r_{1}\right) \mathbb{G}\left(\mathrm{d} r_{2}\right) .
\end{aligned}
$$

Using $d_{B}(u, B(x, r))=d_{B}(u-x, B(\mathrm{o}, r))$ and the reflection invariance of Lebesgue measure, we deduce that

$$
\begin{aligned}
& J_{1}(u)= \gamma \int_{0}^{\infty} \int_{\mathbb{R}^{d}} g\left(d_{B}(x, B(\mathrm{o}, r)), r\right) g\left(d_{B}(u+x, B(\mathrm{o}, r)), r\right) \\
& \times \bar{F}_{B}^{(2)}\left(u ; d_{B}(x, B(\mathrm{o}, r)), d_{B}(u+x, B(\mathrm{o}, r))\right) \mathrm{d} x \mathbb{G}(\mathrm{d} r), \\
& J_{21}(u)=\gamma^{2} \int_{0}^{\infty} \int_{0}^{\infty} \int_{\mathbb{R}^{d}} \int_{\mathbb{R}^{d}} g\left(d_{B}\left(x_{1}, B\left(\mathrm{o}, r_{1}\right)\right), r_{1}\right) g\left(d_{B}\left(x_{2}, B\left(\mathrm{o}, r_{2}\right)\right), r_{2}\right) \\
& \times \mathbf{1}\left\{d_{B}\left(x_{2}, B\left(u, r_{2}\right)\right)>d_{B}\left(x_{1}, B\left(\mathrm{o}, r_{1}\right)\right)\right\} \\
& \times \mathbf{1}\left\{d_{B}\left(x_{1}, B\left(-u, r_{1}\right)\right)>d_{B}\left(x_{2}, B\left(\mathrm{o}, r_{2}\right)\right)\right\} \\
& \times \bar{F}_{B}^{(2)}\left(u ; d_{B}\left(x_{1}, B\left(\mathrm{o}, r_{1}\right)\right), d_{B}\left(x_{2}, B\left(\mathrm{o}, r_{2}\right)\right)\right) \mathrm{d} x_{1} \mathrm{~d} x_{2} \mathbb{G}\left(\mathrm{d} r_{1}\right) \mathbb{G}\left(\mathrm{d} r_{2}\right),
\end{aligned}
$$

and

$$
\begin{aligned}
J_{22}=\gamma^{2} \int_{0}^{\infty} \int_{0}^{\infty} \int_{\mathbb{R}^{d}} \int_{\mathbb{R}^{d}} g\left(d_{B}\left(x_{1}, B\left(\mathrm{o}, r_{1}\right)\right), r_{1}\right) g\left(d_{B}\left(x_{2}, B\left(\mathrm{o}, r_{2}\right)\right), r_{2}\right) \\
\quad \times \bar{F}_{B}\left(d_{B}\left(x_{1}, B\left(\mathrm{o}, r_{1}\right)\right)\right) \bar{F}_{B}\left(d_{B}\left(x_{2}, B\left(\mathrm{o}, r_{2}\right)\right)\right) \mathrm{d} x_{1} \mathrm{~d} x_{2} \mathbb{G}\left(\mathrm{d} r_{1}\right) \mathbb{G}\left(\mathrm{d} r_{2}\right) .
\end{aligned}
$$

The assertion now follows by recalling (2.11). The integrability of $\tau_{1}(C, \cdot)$ and $\tau_{2}(C, \cdot)$, which is explicitly stated in (2.15), will be shown in the proof of Theorem 2.4 and is implied by the assumption (2.8).

Remark 2.3. Recall that $\|\cdot\|$ denotes the Euclidean norm on $\mathbb{R}^{d}$. If $B=B^{d}$ is the unit ball, then $d_{B^{d}}(u, B(x, r))=(\|x-u\|-r)^{+}$,

$$
h_{B^{d}}(t, r)=\sum_{j=0}^{d-1} d \kappa_{d}\left(\begin{array}{c}
d-1 \\
j
\end{array}\right) r^{d-1-j} t^{j}=d \kappa_{d}(t+r)^{d-1},
$$

and

$$
\kappa_{B^{d}}\left(u ; t_{1}, t_{2}, r\right)=\left|B\left(\mathrm{o}, t_{1}+r\right) \cap B\left(u, t_{2}+r\right)\right|_{d} .
$$


Hence, $\tau_{1}(C, u)$ and $\tau_{2}(C, u)$ from Proposition 2.2 may be slightly simplified. In particular, then we have

$$
\begin{aligned}
\tau_{2}(C, u)=\int_{C} & \int_{C} \int_{\mathbb{R}^{d}} \int_{\mathbb{R}^{d}} \frac{f\left(\left(\left\|x_{1}\right\|-r_{1}\right)^{+}\right)}{h_{B^{d}}\left(\left(\left\|x_{1}\right\|-r_{1}\right)^{+}, r_{1}\right)} \frac{f\left(\left(\left\|x_{2}\right\|-r_{2}\right)^{+}\right)}{h_{B^{d}}\left(\left(\left\|x_{2}\right\|-r_{2}\right)^{+}, r_{2}\right)} \\
& \times\left[\mathbf{1}\left\{\left(\left\|x_{2}-u\right\|-r_{2}\right)^{+}>\left(\left\|x_{1}\right\|-r_{1}\right)^{+}\right\} \mathbf{1}\left\{\left(\left\|x_{1}+u\right\|-r_{1}\right)^{+}>\left(\left\|x_{2}\right\|-r_{2}\right)^{+}\right\}\right. \\
& \left.\times \bar{F}_{B^{d}}^{(2)}\left(u ;\left(\left\|x_{1}\right\|-r_{1}\right)^{+},\left(\left\|x_{2}\right\|-r_{2}\right)^{+}\right)-\bar{F}_{B^{d}}\left(\left(\left\|x_{1}\right\|-r_{1}\right)^{+}\right) \bar{F}_{B^{d}}\left(\left(\left\|x_{2}\right\|-r_{2}\right)^{+}\right)\right] \\
& \times \mathrm{d} x_{1} \mathrm{~d} x_{2} \mathbb{G}\left(\mathrm{d} r_{1}\right) \mathbb{G}\left(\mathrm{d} r_{2}\right) \\
= & \int_{C} \int_{C} \int_{0}^{\infty} \int_{\mathbb{S}^{d-1}} \int_{0}^{\infty} \int_{\mathbb{S}^{d-1}} \frac{f\left(s_{1}\right)}{h_{B^{d}}\left(s_{1}, r_{1}\right)}\left(s_{1}+r_{1}\right)^{d-1} \frac{f\left(s_{2}\right)}{h_{B^{d}}\left(s_{2}, r_{2}\right)}\left(s_{2}+r_{2}\right)^{d-1} \\
& \times\left[\mathbf{1}\left\{\left(\left\|\left(s_{2}+r_{2}\right) v_{2}-u\right\|-r_{2}\right)^{+}>s_{1}\right\} \mathbf{1}\left\{\left(\left\|\left(s_{1}+r_{1}\right) v_{1}+u\right\|-r_{1}\right)^{+}>s_{2}\right\}\right. \\
& \left.\times \bar{F}_{B^{d}}^{(2)}\left(u ; s_{1}, s_{2}\right)-\bar{F}_{B^{d}}\left(s_{1}\right) \bar{F}_{B^{d}}\left(s_{2}\right)\right] \mathcal{H}^{d-1}\left(\mathrm{~d} v_{1}\right) \mathrm{d} s_{1} \mathcal{H}^{d-1}\left(\mathrm{~d} v_{2}\right) \mathrm{d} s_{2} \mathbb{G}_{(}\left(\mathrm{d} r_{1}\right) \mathbb{G}\left(\mathrm{d} r_{2}\right) \\
= & \int_{C} \int_{C}^{\infty} \int_{0}^{\infty} f\left(s_{1}\right) f\left(s_{2}\right)\left[\frac{\mathcal{H}^{d-1}\left(\partial B\left(\mathrm{o}, s_{2}+r_{2}\right) \cap B\left(u, s_{1}+r_{2}\right)^{c}\right)}{\mathcal{H}^{d-1}\left(\partial B\left(\mathrm{o}, s_{2}+r_{2}\right)\right)}\right. \\
& \left.\times \frac{\mathcal{H}^{d-1}\left(\partial B\left(\mathrm{o}, s_{1}+r_{1}\right) \cap B\left(-u, s_{2}+r_{1}\right)^{c}\right)}{\mathcal{H}^{d-1}\left(\partial B\left(\mathrm{o}, s_{1}+r_{1}\right)\right)} \bar{F}_{B^{d}}^{(2)}\left(u ; s_{1}, s_{2}\right)-\bar{F}_{B^{d}}\left(s_{1}\right) \bar{F}_{B^{d}}\left(s_{2}\right)\right] \\
& \times \mathrm{d} s_{1} \mathrm{~d} s_{2} \mathbb{G}\left(\mathrm{d} r_{1}\right) \mathbb{G}\left(\mathrm{d} r_{2}\right),
\end{aligned}
$$

where $\mathbb{S}^{d-1}$ is the unit sphere in $\mathbb{R}^{d}, \mathcal{H}^{d-1}$ is the $(d-1)$-dimensional Hausdorff measure, and $\partial B(x, r)$ is the boundary of $B(x, r)$. We used that $f\left((\|x\|-r)^{+}\right)$is non-zero only if $\|x\|>r$. Then $x=(s+r) v$ for $s>0$ and $v \in \mathbb{S}^{d-1}$.

Next we state a special case of [10, Theorem 2.1 and Remark 3.1] in the form needed in the present context. Let $\tilde{g}: \mathbb{R}^{d} \rightarrow[0, \infty]$ be measurable, and let $K, B \subset \mathbb{R}^{d}$ be convex bodies such that $\mathrm{o} \in B$ and $K, B$ are in general relative position. Since in our application, we shall only need the case $K=r B^{d}$, for $r \in \mathbb{R}^{+}$, the assumption of general relative position will be satisfied for any choice of $B$. Then we have

$$
\begin{aligned}
\int_{\mathbb{R}^{d}} \mathbf{1} & \left\{0<d_{B}\left(z, r B^{d}\right)<\infty\right\} \tilde{g}(z) \mathrm{d} z \\
= & \sum_{j=0}^{d-1}\left(\begin{array}{c}
d-1 \\
j
\end{array}\right) \int_{0}^{\infty} \int t^{d-1-j} \tilde{g}(z+t b) \Theta_{j ; d-j}\left(r B^{d} ; B^{*} ; \mathrm{d}(z, b)\right) \mathrm{d} t
\end{aligned}
$$

where $B^{*}:=-B$ and the mixed support measures $\Theta_{j ; d-j}\left(r B^{d} ; B^{*} ; \cdot\right), j \in\{0, \ldots, d-1\}$, are finite Borel measures on $\mathbb{R}^{2 d}$. Using [20, (14.18)] (cf. [18, (4.2.26) and (5.3.8)]) and [12, middle of p. 327], we obtain for the total measures $\Theta_{j ; d-j}\left(r B^{d} ; B^{*} ; \mathbb{R}^{2 d}\right)=r^{j} d\left(\begin{array}{l}d \\ j\end{array}\right) \kappa_{j} V_{d-j}(B)$. In particular, this yields for any measurable function $\tilde{f}:[0, \infty] \rightarrow[0, \infty]$ with $\tilde{f}(0)=\tilde{f}(\infty)=0$ that

$$
\int_{\mathbb{R}^{d}} \tilde{f}\left(d_{B}\left(z, r B^{d}\right)\right) \mathrm{d} z=\int_{0}^{\infty} h_{B}(t, r) \tilde{f}(t) \mathrm{d} t .
$$

We now turn to the asymptotic properties of the ratio-unbiased estimator 1.8 . Our setting is similar to [15], where all limit theorems refer to a growing observation window in $\mathbb{R}^{d}$. More formally, we 
consider a sequence $\left(W_{n}\right)_{n \in \mathbb{N}}$ of compact, convex sets $W_{n} \subset \mathbb{R}^{d}$ such that $W_{n} \subset W_{n+1}$ for all $n \in \mathbb{N}$ and the inradius of $W_{n}$ tends to $\infty$ as $n \rightarrow \infty$.

Theorem 2.4. Assume that (2.8) is fulfilled. Then

$$
\frac{\operatorname{Var} \eta_{W_{n}}(C)}{\left|W_{n}\right|_{d}} \underset{n \rightarrow \infty}{\longrightarrow} \sigma^{2}(C)
$$

The asymptotic variance is finite and given by

$$
\sigma^{2}(C)=\gamma \int_{\mathbb{R}^{d}} \tau_{1}(C, u) \mathrm{d} u+\gamma^{2} \int_{\mathbb{R}^{d}} \tau_{2}(C, u) \mathrm{d} u,
$$

where $\tau_{1}(C, u)$ and $\tau_{2}(C, u)$ are defined in (2.9) and (2.10), respectively. Moreover, if $0<\mathbb{G}(C)<1$, then $\sigma^{2}(C)>0$.

Proof. Suppose that $x \in W_{n}$ and $u \in \mathbb{R}^{d}$. If $x+u \notin W_{n}$, then $d_{B^{d}}\left(x, \partial W_{n}\right) \leq\|u\|$. Hence, we obtain

$$
\left|W_{n}\right|_{d}-\left|\left\{x \in W_{n}: d_{B^{d}}\left(x, \partial W_{n}\right) \leq\|u\|\right\}\right|_{d} \leq\left|W_{n} \cap\left(W_{n}-u\right)\right|_{d} \leq\left|W_{n}\right|_{d} .
$$

Thus, [11, Lemma 10.15 (ii)] implies that

$$
\frac{\left|W_{n} \cap\left(W_{n}-u\right)\right|_{d}}{\left|W_{n}\right|_{d}} \underset{n \rightarrow \infty}{\longrightarrow} 1 \text { for any } u \in \mathbb{R}^{d}
$$

Therefore Lebesgue's dominated convergence theorem and Proposition 2.2 yield 2.13 provided that

$$
\int_{\mathbb{R}^{d}} \tau_{1}(C, u) \mathrm{d} u<\infty \quad \text { and } \quad \int_{\mathbb{R}^{d}}\left|\tau_{2}(C, u)\right| \mathrm{d} u<\infty .
$$

Using (2.7) we have

$$
\begin{aligned}
\int_{\mathbb{R}^{d}} \tau_{1}(C, u) \mathrm{d} u \leq & \int_{C} \int_{\mathbb{R}^{d}} \int_{\mathbb{R}^{d}} \frac{f\left(d_{B}\left(x, r B^{d}\right)\right)}{h_{B}\left(d_{B}\left(x, r B^{d}\right), r\right)} \frac{f\left(d_{B}\left(y, r B^{d}\right)\right)}{h_{B}\left(d_{B}\left(y, r B^{d}\right), r\right)} \\
& \times \sqrt{\bar{F}_{B}\left(d_{B}\left(x, r B^{d}\right)\right) \bar{F}_{B}\left(d_{B}\left(y, r B^{d}\right)\right)} \mathrm{d} x \mathrm{~d} y \mathbb{G}(\mathrm{d} r) \\
= & \int_{C}\left(\int_{\mathbb{R}^{d}} \frac{f\left(d_{B}\left(x, r B^{d}\right)\right)}{h_{B}\left(d_{B}\left(x, r B^{d}\right), r\right)} \sqrt{\bar{F}_{B}\left(d_{B}\left(x, r B^{d}\right)\right)} \mathrm{d} x\right)^{2} \mathbb{G}(\mathrm{d} r) .
\end{aligned}
$$

An application of (2.12) shows that

$$
\int_{\mathbb{R}^{d}} \frac{f\left(d_{B}\left(x, r B^{d}\right)\right)}{h_{B}\left(d_{B}\left(x, r B^{d}\right), r\right)} \sqrt{\bar{F}_{B}\left(d_{B}\left(x, r B^{d}\right)\right)} \mathrm{d} x=\int_{0}^{\infty} f(t) \sqrt{\bar{F}_{B}(t)} \mathrm{d} t
$$

and thus we obtain

$$
\begin{aligned}
\int_{\mathbb{R}^{d}} \tau_{1}(C, u) \mathrm{d} u & \leq \int_{C}\left(\int_{0}^{\infty} f(t) \sqrt{\bar{F}_{B}(t)} \mathrm{d} t\right)^{2} \mathbb{G}(\mathrm{d} r) \\
& \leq \mathbb{G}(C)\left(\int_{0}^{\infty} f(t) \mathrm{e}^{-2 c t} \mathrm{~d} t\right)^{2}<\infty
\end{aligned}
$$

where we use that $\bar{F}_{B}(t) \leq \exp \{-4 c t\}$ and assumption (2.8). 
In order to show that

$$
\int_{\mathbb{R}^{d}}\left|\tau_{2}(C, u)\right| \mathrm{d} u<\infty
$$

we first rewrite $q\left(u ; x_{1}, x_{2}, r_{1}, r_{2}\right)$ as the difference of two non-negative terms, that is, $q=q_{1}-q_{2}$ with

$$
\begin{aligned}
q_{1}\left(u ; x_{1}, x_{2}, r_{1}, r_{2}\right):= & \bar{F}_{B}^{(2)}\left(u ; d_{B}\left(x_{1}, B\left(\mathrm{o}, r_{1}\right)\right), d_{B}\left(x_{2}, B\left(\mathrm{o}, r_{2}\right)\right)\right) \\
& -\bar{F}_{B}\left(d_{B}\left(x_{1}, B\left(\mathrm{o}, r_{1}\right)\right)\right) \bar{F}_{B}\left(d_{B}\left(x_{2}, B\left(\mathrm{o}, r_{2}\right)\right)\right),
\end{aligned}
$$

which is non-negative by (2.6), and

$$
\begin{aligned}
& q_{2}\left(u ; x_{1}, x_{2}, r_{1}, r_{2}\right):=\bar{F}_{B}^{(2)}\left(u ; d_{B}\left(x_{1}, B\left(\mathrm{o}, r_{1}\right)\right), d_{B}\left(x_{2}, B\left(\mathrm{o}, r_{2}\right)\right)\right) \\
& \quad \times\left(1-\mathbf{1}\left\{d_{B}\left(x_{2}, B\left(u, r_{2}\right)\right)>d_{B}\left(x_{1}, B\left(\mathrm{o}, r_{1}\right)\right)\right\} \mathbf{1}\left\{d_{B}\left(x_{1}, B\left(-u, r_{1}\right)\right)>d_{B}\left(x_{2}, B\left(\mathrm{o}, r_{2}\right)\right)\right\}\right),
\end{aligned}
$$

for $u, x_{1}, x_{2} \in \mathbb{R}^{d}$ and $r_{1}, r_{2} \in \mathbb{R}^{+}$. Using (2.4), (2.7) and the inequality $1-\mathrm{e}^{-a} \leq a$, for $a \geq 0$, we get

$$
\begin{aligned}
& q_{1}\left(u ; x_{1}, x_{2}, r_{1}, r_{2}\right) \leq \bar{F}_{B}^{(2)}\left(u ; d_{B}\left(x_{1}, B\left(\mathrm{o}, r_{1}\right)\right), d_{B}\left(x_{2}, B\left(\mathrm{o}, r_{2}\right)\right)\right) \\
& \times\left(1-\exp \left\{-\gamma \mathbb{E} \kappa_{B}\left(u ; d_{B}\left(x_{1}, B\left(\mathrm{o}, r_{1}\right)\right), d_{B}\left(x_{2}, B\left(\mathrm{o}, r_{2}\right)\right), R\right)\right\}\right) \\
& \leq \sqrt{\bar{F}_{B}\left(d_{B}\left(x_{1}, B\left(\mathrm{o}, r_{1}\right)\right)\right) \bar{F}_{B}\left(d_{B}\left(x_{2}, B\left(\mathrm{o}, r_{2}\right)\right)\right)} \\
& \times \gamma \mathbb{E} \kappa_{B}\left(u ; d_{B}\left(x_{1}, B\left(\mathrm{o}, r_{1}\right)\right), d_{B}\left(x_{2}, B\left(\mathrm{o}, r_{2}\right)\right), R\right) .
\end{aligned}
$$

Moreover, the inequality $1-(1-a)(1-b) \leq a+b$, for $a, b \geq 0$, and again (2.7) imply that

$$
\begin{aligned}
& q_{2}\left(u ; x_{1}, x_{2}, r_{1}, r_{2}\right) \leq \sqrt{\bar{F}_{B}\left(d_{B}\left(x_{1}, B\left(\mathrm{o}, r_{1}\right)\right)\right) \bar{F}_{B}\left(d_{B}\left(x_{2}, B\left(\mathrm{o}, r_{2}\right)\right)\right)} \\
& \times\left(\mathbf{1}\left\{d_{B}\left(x_{2}, B\left(u, r_{2}\right)\right) \leq d_{B}\left(x_{1}, B\left(\mathrm{o}, r_{1}\right)\right)\right\}\right. \\
& \left.+\mathbf{1}\left\{d_{B}\left(x_{1}, B\left(-u, r_{1}\right)\right) \leq d_{B}\left(x_{2}, B\left(\mathrm{o}, r_{2}\right)\right)\right\}\right) .
\end{aligned}
$$

Combining these bounds, we arrive at

$$
\begin{aligned}
\int_{\mathbb{R}^{d}}\left|\tau_{2}(C, u)\right| \mathrm{d} u \leq \int_{C} \int_{C} \int_{\mathbb{R}^{d}} \int_{\mathbb{R}^{d}} \int_{\mathbb{R}^{d}} \frac{f\left(d_{B}\left(x_{1}, B\left(\mathrm{o}, r_{1}\right)\right)\right)}{h_{B}\left(d_{B}\left(x_{1}, B\left(\mathrm{o}, r_{1}\right)\right), r_{1}\right)} \frac{f\left(d_{B}\left(x_{2}, B\left(\mathrm{o}, r_{2}\right)\right)\right)}{h_{B}\left(d_{B}\left(x_{2}, B\left(\mathrm{o}, r_{2}\right)\right), r_{2}\right)} \\
\times \sqrt{\bar{F}_{B}\left(d_{B}\left(x_{1}, B\left(\mathrm{o}, r_{1}\right)\right)\right) \bar{F}_{B}\left(d_{B}\left(x_{2}, B\left(\mathrm{o}, r_{2}\right)\right)\right)} \\
\times\left[\gamma \mathbb{E} \kappa_{B}\left(u ; d_{B}\left(x_{1}, B\left(\mathrm{o}, r_{1}\right)\right), d_{B}\left(x_{2}, B\left(\mathrm{o}, r_{2}\right)\right), R\right)\right. \\
\quad+\mathbf{1}\left\{d_{B}\left(x_{2}, B\left(u, r_{2}\right)\right) \leq d_{B}\left(x_{1}, B\left(\mathrm{o}, r_{1}\right)\right)\right\} \\
\left.\quad+\mathbf{1}\left\{d_{B}\left(x_{1}, B\left(-u, r_{1}\right)\right) \leq d_{B}\left(x_{2}, B\left(\mathrm{o}, r_{2}\right)\right)\right\}\right] \\
\times \mathrm{d} x_{1} \mathrm{~d} x_{2} \mathrm{~d} u \mathbb{G}\left(\mathrm{d} r_{1}\right) \mathbb{G}\left(\mathrm{d} r_{2}\right) .
\end{aligned}
$$

The preceding expression splits naturally into three summands which will be bounded from above separately. For the first bound, we observe that by Fubini's theorem

$$
\mathbb{E} \int_{\mathbb{R}^{d}} \kappa_{B}\left(u ; s_{1}, s_{2}, R\right) \mathrm{d} u=\mathbb{E}\left|B_{s_{1}, R}\right|_{d}\left|B_{s_{2}, R}\right|_{d}
$$


Then we apply (2.12) to get

$$
\begin{aligned}
& \int_{C} \int_{C} \int_{\mathbb{R}^{d}} \int_{\mathbb{R}^{d}} \int_{\mathbb{R}^{d}} \frac{f\left(d_{B}\left(x_{1}, B\left(\mathrm{o}, r_{1}\right)\right)\right)}{h_{B}\left(d_{B}\left(x_{1}, B\left(\mathrm{o}, r_{1}\right)\right), r_{1}\right)} \frac{f\left(d_{B}\left(x_{2}, B\left(\mathrm{o}, r_{2}\right)\right)\right)}{h_{B}\left(d_{B}\left(x_{2}, B\left(\mathrm{o}, r_{2}\right)\right), r_{2}\right)} \\
& \times \sqrt{\bar{F}_{B}\left(d_{B}\left(x_{1}, B\left(\mathrm{o}, r_{1}\right)\right)\right) \bar{F}_{B}\left(d_{B}\left(x_{2}, B\left(\mathrm{o}, r_{2}\right)\right)\right)} \\
& \times \gamma \mathbb{E} \kappa_{B}\left(u ; d_{B}\left(x_{1}, B\left(\mathrm{o}, r_{1}\right)\right), d_{B}\left(x_{2}, B\left(\mathrm{o}, r_{2}\right)\right), R\right) \\
& \times \mathrm{d} x_{1} \mathrm{~d} x_{2} \mathrm{~d} u \mathbb{G}\left(\mathrm{d} r_{1}\right) \mathbb{G}\left(\mathrm{d} r_{2}\right) \\
&=\gamma \mathbb{G}(C)^{2} \int_{0}^{\infty} \int_{0}^{\infty} f\left(t_{1}\right) \sqrt{\bar{F}_{B}\left(t_{1}\right)} f\left(t_{2}\right) \sqrt{\bar{F}_{B}\left(t_{2}\right)} \mathbb{E}\left|B_{t_{1}, R}\right|_{d}\left|B_{t_{2}, R}\right|_{d} \mathrm{~d} t_{1} \mathrm{~d} t_{2} .
\end{aligned}
$$

Choose $c_{B}>0$ such that $B \subset c_{B} B^{d}$. Then $\left|B_{t, R}\right|_{d} \leq \kappa_{d}\left(c_{B} t+R\right)^{d}$ and hence the Cauchy-Schwarz inequality, the convexity of $s \mapsto s^{p}, p \geq 1$, and $\sqrt{a+b} \leq \sqrt{a}+\sqrt{b}, a, b \geq 0$, yield that

$$
\begin{aligned}
\mathbb{E}\left|B_{t_{1}, R}\right|_{d}\left|B_{t_{2}, R}\right|_{d} & \leq c_{1} \sqrt{\mathbb{E}\left(c_{B} t_{1}+R\right)^{2 d}} \sqrt{\mathbb{E}\left(c_{B} t_{2}+R\right)^{2 d}} \\
& \leq c_{2}\left(c_{B}^{d} t_{1}^{d}+\sqrt{\mathbb{E} R^{2 d}}\right)\left(c_{B}^{d} t_{2}^{d}+\sqrt{\mathbb{E} R^{2 d}}\right) \\
& \leq c_{3}\left(1+t_{1}^{d}\right)\left(1+t_{2}^{d}\right),
\end{aligned}
$$

where $c_{1}, c_{2}, c_{3}$ denote finite constants independent of the expectation or $t_{1}, t_{2}$. From this and (2.8) it follows again that the first summand is finite.

Since $d_{B}\left(x_{2}, B\left(u, r_{2}\right)\right) \leq t_{1}$ if and only if $u \in x_{2}+B_{t_{1}, r_{2}}$, applying Fubini's theorem and (2.12) (twice) we obtain for the second summand that

$$
\begin{aligned}
& \int_{C} \int_{C} \int_{\mathbb{R}^{d}} \int_{\mathbb{R}^{d}} \int_{\mathbb{R}^{d}} \frac{f\left(d_{B}\left(x_{1}, B\left(\mathrm{o}, r_{1}\right)\right)\right)}{h_{B}\left(d_{B}\left(x_{1}, B\left(\mathrm{o}, r_{1}\right)\right), r_{1}\right)} \frac{f\left(d_{B}\left(x_{2}, B\left(\mathrm{o}, r_{2}\right)\right)\right)}{h_{B}\left(d_{B}\left(x_{2}, B\left(\mathrm{o}, r_{2}\right)\right), r_{2}\right)} \\
& \quad \times \sqrt{\bar{F}_{B}\left(d_{B}\left(x_{1}, B\left(\mathrm{o}, r_{1}\right)\right)\right) \bar{F}_{B}\left(d_{B}\left(x_{2}, B\left(\mathrm{o}, r_{2}\right)\right)\right)} \\
& \quad \times \mathbf{1}\left\{d_{B}\left(x_{2}, B\left(u, r_{2}\right)\right) \leq d_{B}\left(x_{1}, B\left(\mathrm{o}, r_{1}\right)\right)\right\} \\
& \quad \times \mathrm{d} x_{1} \mathrm{~d} x_{2} \mathrm{~d} u \mathbb{G}\left(\mathrm{d} r_{1}\right) \mathbb{G}\left(\mathrm{d} r_{2}\right) \\
& =\int_{C} \int_{C} \int_{0}^{\infty} \int_{0}^{\infty} f\left(t_{1}\right) \sqrt{\bar{F}_{B}\left(t_{1}\right)} f\left(t_{2}\right) \sqrt{\bar{F}_{B}\left(t_{2}\right)}\left|B_{t_{1}, r_{2}}\right|_{d} \mathrm{~d} t_{1} \mathrm{~d} t_{2} \mathbb{G}\left(\mathrm{d} r_{1}\right) \mathbb{G}\left(\mathrm{d} r_{2}\right),
\end{aligned}
$$

which is finite by the same reasoning as above.

The third summand can be treated in exactly the same way.

To prove positivity of the asymptotic variance we use the fact that the variance of any squareintegrable function $H(\Psi)$ of the Poisson process $\Psi:=\left\{\left(\xi_{n}, R_{n}\right): n \geq 1\right\}$ satisfies the inequality

$$
\operatorname{Var} H(\Psi) \geq \gamma \int_{0}^{\infty} \int_{\mathbb{R}^{d}}(\mathbb{E}[H(\Psi \cup\{(y, r)\})-H(\Psi)])^{2} \mathrm{~d} y \mathbb{G}(\mathrm{d} r) ;
$$

see, e.g., [13, Theorem 4.2]. In our case this means that

$$
\operatorname{Var} \eta_{W}(C) \geq \gamma \int_{0}^{\infty} \int_{\mathbb{R}^{d}} \tilde{h}(y, r)^{2} \mathrm{~d} y \mathbb{G}(\mathrm{d} r),
$$


where

$$
\begin{aligned}
\tilde{h}(y, r):=\mathbb{E} \int_{W}\left[g\left(d_{B}(x, Z \cup B(y, r)), r_{B}(x, Z \cup B(y, r))\right)-g\left(d_{B}(x, Z), r_{B}(x, Z)\right)\right] \mathrm{d} x \\
=\mathbb{E} \int_{W} \mathbf{1}\left\{d_{B}(x, B(y, r))<d_{B}(x, Z)\right\}\left[g\left(d_{B}(x, B(y, r)), r\right)-g\left(d_{B}(x, Z), r_{B}(x, Z)\right)\right] \mathrm{d} x \\
=\mathbb{E} \int_{W} \mathbf{1}\left\{d_{B}(\mathrm{o}, B(y-x, r))<d_{B}(\mathrm{o}, Z)\right\} \\
\times\left[g\left(d_{B}(\mathrm{o}, B(y-x, r)), r\right)-g\left(d_{B}(\mathrm{o}, Z), r_{B}(\mathrm{o}, Z)\right)\right] \mathrm{d} x .
\end{aligned}
$$

Here the last identity follows from the stationarity of $Z$ and $g$ is as defined in (2.11). By (1.4),

$$
\begin{aligned}
\tilde{h}(y, r)=\gamma \int_{W} & \int_{0}^{\infty} \int_{0}^{\infty} \mathbf{1}\left\{d_{B}(\mathrm{o}, B(y-x, r))<t\right\} \\
& \times\left[g\left(d_{B}(\mathrm{o}, B(y-x, r)), r\right)-g(t, s)\right] h_{B}(t, s) \bar{F}_{B}(t) \mathrm{d} t \mathbb{G}(\mathrm{d} s) \mathrm{d} x .
\end{aligned}
$$

Assume now that $0<\mathbb{G}(C)<1$ and let $C^{\prime}:=\mathbb{R}^{+} \backslash C$. Recalling the definition (2.11) of $g$, we obtain from (2.16) that

$$
\operatorname{Var} \eta_{W}(C) \geq \gamma \int_{0}^{\infty} \int_{\mathbb{R}^{d}} h^{*}(y, r)^{2} \mathbf{1}\left\{r \in C^{\prime}, y \in W\right\} \mathrm{d} y \mathbb{G}(\mathrm{d} r)
$$

where

$$
\begin{aligned}
h^{*}(y, r) & :=\gamma \int_{W} \int_{0}^{\infty} \int_{0}^{\infty} \mathbf{1}\left\{d_{B}(\mathrm{o}, B(y-x, r))<t\right\} g(t, s) h_{B}(t, s) \bar{F}_{B}(t) \mathrm{d} t \mathbb{G}(\mathrm{d} s) \mathrm{d} x \\
& =\gamma \mathbb{G}(C) \int_{W} \int_{0}^{\infty} \mathbf{1}\left\{d_{B}(\mathrm{o}, B(y-x, r))<t\right\} f(t) \bar{F}_{B}(t) \mathrm{d} t \mathrm{~d} x .
\end{aligned}
$$

Applying Jensen's inequality with the normalization of $\mathbf{1}\left\{r \in C^{\prime}, y \in W\right\} \mathrm{d} y \mathbb{G}(\mathrm{d} r)$, we get

$$
\operatorname{Var} \eta_{W}(C) \geq \frac{\gamma}{\mathbb{G}\left(C^{\prime}\right)|W|_{d}}\left(\int_{C^{\prime}} \int_{W} h^{*}(y, r) \mathrm{d} y \mathbb{G}(\mathrm{d} r)\right)^{2} .
$$

Letting $a:=\gamma^{3} \mathbb{G}(C)^{2} / \mathbb{G}\left(C^{\prime}\right)>0$ we obtain that

$$
\begin{aligned}
& \frac{\operatorname{Var} \eta_{W}(C)}{|W|_{d}} \\
& \geq \frac{a}{|W|_{d}^{2}}\left(\int_{C^{\prime}} \int_{W} \int_{W} \int_{0}^{\infty} \mathbf{1}\left\{d_{B}(\mathrm{o}, B(y-x, r))<t\right\} f(t) \bar{F}_{B}(t) \mathrm{d} t \mathrm{~d} x \mathrm{~d} y \mathbb{G}(\mathrm{d} r)\right)^{2} \\
& \quad=\frac{a}{|W|_{d}^{2}}\left(\int_{C^{\prime}} \int_{\mathbb{R}^{d}} \int_{0}^{\infty}|W \cap(W-y)|_{d} \mathbf{1}\left\{d_{B}(\mathrm{o}, B(y, r))<t\right\} f(t) \bar{F}_{B}(t) \mathrm{d} t \mathrm{~d} y \mathbb{G}(\mathrm{d} r)\right)^{2} .
\end{aligned}
$$

Hence it is sufficient to show that

$$
\int_{C^{\prime}} \int_{0}^{\infty}\left(\int_{\mathbb{R}^{d}} \mathbf{1}\left\{d_{B}(\mathrm{o}, B(y, r))<t\right\} \mathrm{d} y\right) f(t) \bar{F}_{B}(t) \mathrm{d} t \mathbb{G}(\mathrm{d} r)>0 .
$$

This is true, since the inner integral is positive for all $r, t>0$ and since both $\int_{0}^{\infty} f(t) \bar{F}_{B}(t) \mathrm{d} t$ and $\mathbb{G}\left(C^{\prime}\right)$ are positive. 
Remark 2.5. The assumption (2.8) is slightly stronger than (1.6).

Remark 2.6. Let $\widehat{\mathbb{G}}_{n}(C)$ be given by $(1.8)$ with $W=W_{n}$. Theorem 2.4 implies that $\widehat{\mathbb{G}}_{n}(C)$ is asymptotically weakly consistent. Indeed, (1.7) and

$$
\frac{\operatorname{Var} \eta_{W_{n}}(C)}{\left|W_{n}\right|_{d}^{2}} \underset{n \rightarrow \infty}{\longrightarrow} 0
$$

ensure that $\eta_{W_{n}}(C) /\left|W_{n}\right|_{d}$ converges to $\gamma \beta \mathbb{G}(C)$ in probability as $n \rightarrow \infty$. Especially,

$$
\frac{\eta_{W_{n}}\left(\mathbb{R}^{+}\right)}{\left|W_{n}\right|_{d}} \underset{n \rightarrow \infty}{\longrightarrow} \gamma \beta \quad \text { in probability }
$$

Hence, by the continuous mapping theorem, $\eta_{W_{n}}(C) / \eta_{W}\left(\mathbb{R}^{+}\right)$converges to $\mathbb{G}(C)$ in probability as $n \rightarrow$ $\infty$. This is in accordance with the following proposition which even shows that $\widehat{\mathbb{G}}_{n}(C)$ is asymptotically strongly consistent.

Proposition 2.7. For any Borel set $C \subset \mathbb{R}^{+}$, we have $\widehat{\mathbb{G}}_{n}(C) \underset{n \rightarrow \infty}{\longrightarrow} \mathbb{G}(C) \mathbb{P}$-a.s.

Proof. The mapping $W \mapsto \eta_{W}(C)$ defined by (1.5) is a random measure on $\mathbb{R}^{d}$ depending on the Boolean model $Z$ in a translation-invariant way. As the Boolean model is ergodic (see [20, Theorem 9.3.5]) we can apply the spatial ergodic theorem (see [11, Corollary 10.19]) to conclude that

$$
\lim _{n \rightarrow \infty}\left|W_{n}\right|_{d}^{-1} \eta_{W_{n}}(C)=\mathbb{E} \eta_{[0,1]^{d}}(C)=\gamma \beta \mathbb{G}(C) \quad \mathbb{P} \text {-a.s. }
$$

Applying this to the numerator as well as to the denominator in (1.8), we obtain the desired result.

\section{Asymptotic normality}

In this section we study the asymptotic normality of the ratio-unbiased estimator (1.8) for the radius distribution $\mathbb{G}$ of our stationary Boolean model $Z$ with spherical grains. The proof will be based on approximation by $m$-dependent random fields. This idea comes from [7], where the same technique was used to prove the central limit theorem for random measures which are associated with the Boolean model in an additive way. In contrast to [7], the contribution of an individual grain to the random measure $A \mapsto \eta_{A}(C)$ is not determined by the grain alone, but does depend on a random number of other grains in a non-trivial manner. Therefore the results of [7] do not apply in our setting.

We consider, for $n \in \mathbb{N}$ and a Borel set $C \subset \mathbb{R}^{+}$, the estimator

$$
\widehat{\mathbb{G}}_{n}(C)=\frac{\eta_{W_{n}}(C)}{\eta_{W_{n}}\left(\mathbb{R}^{d}\right)},
$$

where $W_{n}:=[-n, n)^{d}$ and $\eta_{W_{n}}$ is given in (1.5). First we concentrate on the asymptotic normality of the numerator $\eta_{W_{n}}(C)$. In addition to (1.6), we shall need the integrability condition

$$
\int_{0}^{\infty}\left(1+t^{d}\right) f(t) \mathrm{d} t<\infty
$$

which is more restrictive than (2.8).

Theorem 3.1. Assume that (1.6) and (3.1) are fulfilled. Then, for any Borel set $C \subset \mathbb{R}^{+}$,

$$
\sqrt{\left|W_{n}\right|_{d}}\left(\frac{\eta_{W_{n}}(C)}{\left|W_{n}\right|_{d}}-\gamma \beta \mathbb{G}(C)\right) \underset{n \rightarrow \infty}{\stackrel{\mathrm{d}}{\longrightarrow}} N\left(0, \sigma^{2}(C)\right),
$$

where $\sigma^{2}(C)$ is given by (2.14). 
Proof. We fix a Borel set $C \subset \mathbb{R}^{+}$and skip the dependence on $C$ in the notation. Let $E_{z}:=[0,1)^{d}+z$ for $z \in I_{n}:=\{-n, \ldots, n-1\}^{d}$. Then

$$
\eta_{W_{n}}=\sum_{z \in I_{n}} \eta_{E_{z}}=\sum_{z \in I_{n}} \int_{E_{z}} g\left(d_{B}(x, Z), r_{B}(x, Z)\right) \mathrm{d} x,
$$

where $g$ is given by (2.11). For some fixed integer $m$, we put $F_{z}:=E_{z} \oplus[-m, m)^{d}$. We decompose $\eta_{E_{z}}$ into two random variables

$$
\eta_{z}^{(m)}:=\int_{E_{z}} g\left(d_{B}\left(x, Z\left(F_{z}\right)\right), r_{B}\left(x, Z\left(F_{z}\right)\right)\right) \mathrm{d} x
$$

and $\tilde{\eta}_{z}^{(m)}:=\eta_{E_{z}}-\eta_{z}^{(m)}$. Let $\eta_{W_{n}}^{(m)}:=\sum_{z \in I_{n}} \eta_{z}^{(m)}$ and $\tilde{\eta}_{W_{n}}^{(m)}:=\sum_{z \in I_{n}} \tilde{\eta}_{z}^{(m)}$ so that $\eta_{W_{n}}=\eta_{W_{n}}^{(m)}+\tilde{\eta}_{W_{n}}^{(m)}$. It is easily seen that $\left\{\eta_{u}^{(m)}: u \in U\right\}$ and $\left\{\eta_{v}^{(m)}: v \in V\right\}$ are independent whenever $U, V \subset \mathbb{Z}^{d}$ are such that $\|u-v\|_{\infty}>2 m$ for each $u \in U$ and $v \in V$. Thus, the random variables $\eta_{z}^{(m)}$, for $z \in \mathbb{Z}^{d}$, constitute a stationary $(2 m)$-dependent random field (cf. [6, Section 4.3.1]). The variance of $\eta_{W_{n}}^{(m)}$ is

$$
\operatorname{Var} \eta_{W_{n}}^{(m)}=\operatorname{Var} \sum_{z \in I_{n}} \eta_{z}^{(m)}=\sum_{z_{1} \in I_{n}} \sum_{z_{2} \in I_{n}} \operatorname{Cov}\left(\eta_{z_{1}}^{(m)}, \eta_{z_{2}}^{(m)}\right)=\sum_{z \in I_{n}-I_{n}} N_{n}(z) \operatorname{Cov}\left(\eta_{\mathrm{o}}^{(m)}, \eta_{z}^{(m)}\right),
$$

where $N_{n}(z)$ is the cardinality of $\left\{\left(z_{1}, z_{2}\right) \in I_{n} \times I_{n}: z_{2}-z_{1}=z\right\}$, which may be bounded by $\left|W_{n}\right|_{d}=(2 n)^{d}$ and $\lim _{n \rightarrow \infty} N_{n}(z) /\left|W_{n}\right|_{d}=1$ for any $z \in \mathbb{Z}^{d}$. We define

$$
\left(\sigma_{n}^{(m)}\right)^{2}:=\frac{\operatorname{Var} \eta_{W_{n}}^{(m)}}{\left|W_{n}\right|_{d}}
$$

Since $\operatorname{Cov}\left(\eta_{\mathrm{o}}^{(m)}, \eta_{z}^{(m)}\right)=0$ for $\|z\|>2 m$, the limit of $\left(\sigma_{n}^{(m)}\right)^{2}$ as $n \rightarrow \infty$ exists and satisfies

$$
\left(\sigma^{(m)}\right)^{2}:=\lim _{n \rightarrow \infty}\left(\sigma_{n}^{(m)}\right)^{2}=\sum_{z \in\{-2 m, \ldots, 2 m\}^{d}} \operatorname{Cov}\left(\eta_{\mathrm{o}}^{(m)}, \eta_{z}^{(m)}\right) .
$$

Next we show that $\mathbb{E}\left(\eta_{\mathrm{o}}^{(m)}\right)^{2}<\infty$. We put $A:=[-m, m+1)^{d}$, hence

$$
\eta_{\mathrm{o}}^{(m)}=\int_{E_{\mathrm{o}}} g\left(d_{B}(x, Z(A)), r_{B}(x, Z(A))\right) \mathrm{d} x .
$$

Proceeding as in the proof of Proposition 2.2 and bounding $\bar{F}_{B}^{A, A}(\cdot)$ as well as $(1-\mathbf{1}\{\cdot\} \mathbf{1}\{\cdot\})$ by 1 , we get

$$
\begin{aligned}
\mathbb{E}\left(\eta_{\mathrm{o}}^{(m)}\right)^{2} \leq & \gamma \int_{E_{\mathrm{o}}} \int_{E_{\mathrm{o}}} \int_{0}^{\infty} \int_{A} g\left(d_{B}\left(y, B\left(x_{1}, r\right)\right), r\right) g\left(d_{B}\left(y, B\left(x_{2}, r\right)\right), r\right) \mathrm{d} y \mathbb{G}(\mathrm{d} r) \mathrm{d} x_{1} \mathrm{~d} x_{2} \\
& +\gamma^{2} \int_{E_{\mathrm{o}}} \int_{E_{\mathrm{o}}} \int_{0}^{\infty} \int_{0}^{\infty} \int_{A} \int_{A} g\left(d_{B}\left(y_{1}, B\left(x_{1}, r_{1}\right)\right), r_{1}\right) \\
& \times g\left(d_{B}\left(y_{2}, B\left(x_{2}, r_{2}\right)\right), r_{2}\right) \mathrm{d} y_{1} \mathrm{~d} y_{2} \mathbb{G}\left(\mathrm{d} r_{1}\right) \mathbb{G}\left(\mathrm{d} r_{2}\right) \mathrm{d} x_{1} \mathrm{~d} x_{2} .
\end{aligned}
$$

The right-hand side increases if $A$ is replaced by $\mathbb{R}^{d}$. Arguing then as in the proof of Theorem 2.4, we obtain

$$
\mathbb{E}\left(\eta_{\mathrm{o}}^{(m)}\right)^{2} \leq \gamma\left|E_{\mathrm{o}}\right|_{d}\left(\int_{0}^{\infty} f(t) \mathrm{d} t\right)^{2} \mathbb{G}(C)+\left(\gamma\left|E_{\mathrm{o}}\right|_{d} \int_{0}^{\infty} f(t) \mathrm{d} t \mathbb{G}(C)\right)^{2}<\infty
$$


Therefore, the central limit theorem for stationary $m$-dependent random fields (see, e.g., [17]) yields that

$$
\frac{1}{\sqrt{\left|W_{n}\right|_{d}}} \sum_{z \in I_{n}}\left(\eta_{z}^{(m)}-\mathbb{E} \eta_{z}^{(m)}\right) \underset{n \rightarrow \infty}{\stackrel{\mathrm{d}}{\longrightarrow}} N\left(0,\left(\sigma^{(m)}\right)^{2}\right) .
$$

In view of [2, Theorem 3.2], it remains to verify that

$$
\lim _{m \rightarrow \infty} \sigma^{(m)}=\sigma(C)
$$

and

$$
\lim _{m \rightarrow \infty} \limsup _{n \rightarrow \infty} \mathbb{P}\left(\frac{1}{\sqrt{\left|W_{n}\right|_{d}}}\left|\sum_{z \in I_{n}}\left(\tilde{\eta}_{z}^{(m)}-\mathbb{E} \tilde{\eta}_{z}^{(m)}\right)\right| \geq \varepsilon\right)=0 \quad \text { for any } \varepsilon>0
$$

Define

$$
\sigma_{n}^{2}:=\frac{\operatorname{Var} \eta_{W_{n}}}{\left|W_{n}\right|_{d}}
$$

Then

$$
\left|\sigma(C)-\sigma^{(m)}\right| \leq\left|\sigma(C)-\sigma_{n}\right|+\left|\sigma_{n}-\sigma_{n}^{(m)}\right|+\left|\sigma_{n}^{(m)}-\sigma^{(m)}\right| .
$$

The first term goes to zero as $n \rightarrow \infty$ by Theorem 2.4 the last term goes to zero as $n \rightarrow \infty$ as well, for any $m \in \mathbb{N}$, by (3.2). By Minkowski's inequality, the middle term can be bounded as

$$
\left|\sigma_{n}-\sigma_{n}^{(m)}\right| \leq \frac{1}{\sqrt{\left|W_{n}\right|_{d}}} \sqrt{\operatorname{Var} \tilde{\eta}_{W_{n}}^{(m)}}
$$

Therefore, (3.3) follows if we can show that

$$
\sup _{n \in \mathbb{N}} \frac{1}{\left|W_{n}\right|_{d}} \operatorname{Var} \tilde{\eta}_{W_{n}}^{(m)} \underset{m \rightarrow \infty}{\longrightarrow} 0 .
$$

By Chebyshev's inequality, (3.5) also implies (3.4). The variance in (3.5) satisfies

$$
\frac{1}{\left|W_{n}\right|_{d}} \operatorname{Var} \sum_{z \in I_{n}} \tilde{\eta}_{z}^{(m)}=\frac{1}{\left|W_{n}\right|_{d}} \sum_{z \in I_{n}-I_{n}} N_{n}(z) \operatorname{Cov}\left(\tilde{\eta}_{\mathrm{o}}^{(m)}, \tilde{\eta}_{z}^{(m)}\right) \leq \sum_{z \in \mathbb{Z}^{d}}\left|\operatorname{Cov}\left(\tilde{\eta}_{\mathrm{o}}^{(m)}, \tilde{\eta}_{z}^{(m)}\right)\right| .
$$

Therefore, the proof will be finished when we show that

$$
\sum_{z \in \mathbb{Z}^{d}}\left|\operatorname{Cov}\left(\tilde{\eta}_{\mathrm{o}}^{(m)}, \tilde{\eta}_{z}^{(m)}\right)\right| \underset{m \rightarrow \infty}{\longrightarrow} 0 .
$$

Consider a fixed $z \in \mathbb{Z}^{d}$. Then the covariance can be written as

$$
\begin{gathered}
\operatorname{Cov}\left(\tilde{\eta}_{\mathrm{o}}^{(m)}, \tilde{\eta}_{z}^{(m)}\right)=\operatorname{Cov}\left(\eta_{E_{\mathrm{o}}}, \eta_{E_{z}}\right)-\operatorname{Cov}\left(\eta_{\mathrm{o}}^{(m)}, \eta_{E_{z}}\right)-\operatorname{Cov}\left(\eta_{E_{\mathrm{o}}}, \eta_{z}^{(m)}\right)+\operatorname{Cov}\left(\eta_{\mathrm{o}}^{(m)}, \eta_{z}^{(m)}\right) \\
=\int_{E_{\mathrm{o}}} \int_{E_{z}}\left[c_{\mathbb{R}^{d}, \mathbb{R}^{d}}\left(x_{1}, x_{2}\right)-c_{F_{\mathrm{o}}, \mathbb{R}^{d}}\left(x_{1}, x_{2}\right)-c_{\mathbb{R}^{d}, F_{z}}\left(x_{1}, x_{2}\right)+c_{F_{\mathrm{o}}, F_{z}}\left(x_{1}, x_{2}\right)\right] \mathrm{d} x_{2} \mathrm{~d} x_{1},
\end{gathered}
$$

where, for Borel sets $A_{1}, A_{2} \subset \mathbb{R}^{d}$ and $x_{1}, x_{2} \in \mathbb{R}^{d}$,

$$
c_{A_{1}, A_{2}}\left(x_{1}, x_{2}\right):=\operatorname{Cov}\left(g\left(d_{B}\left(x_{1}, Z\left(A_{1}\right)\right), r_{B}\left(x_{1}, Z\left(A_{1}\right)\right)\right), g\left(d_{B}\left(x_{2}, Z\left(A_{2}\right)\right), r_{B}\left(x_{2}, Z\left(A_{2}\right)\right)\right)\right)
$$


is expressed in Lemma 2.1 as

$$
\begin{aligned}
c_{A_{1}, A_{2}}\left(x_{1}, x_{2}\right)= & \gamma \int_{0}^{\infty} \int_{\mathbb{R}^{d}} g\left(d_{B}\left(x_{1}, B(y, r)\right), r\right) g\left(d_{B}\left(x_{2}, B(y, r)\right), r\right) I_{1}\left(A_{1}, A_{2}\right) \mathrm{d} y \mathbb{G}(\mathrm{d} r) \\
+ & \gamma^{2} \int_{0}^{\infty} \int_{0}^{\infty} \int_{\mathbb{R}^{d}} \int_{\mathbb{R}^{d}} g\left(d_{B}\left(x_{1}, B\left(y_{1}, r_{1}\right)\right), r_{1}\right) g\left(d_{B}\left(x_{2}, B\left(y_{2}, r_{2}\right)\right), r_{2}\right) \\
& \times I_{2}\left(A_{1}, A_{2}\right) \mathrm{d} y_{1} \mathrm{~d} y_{2} \mathbb{G}\left(\mathrm{d} r_{1}\right) \mathbb{G}\left(\mathrm{d} r_{2}\right) .
\end{aligned}
$$

Here we skip the arguments $x_{1}, x_{2}, y, r$, respectively $x_{1}, x_{2}, y_{1}, y_{2}, r_{1}, r_{2}$, of the functions $I_{1}\left(A_{1}, A_{2}\right)$ and $I_{2}\left(A_{1}, A_{2}\right)$, which were defined before Lemma 2.1. We shall treat both parts of $c_{A_{1}, A_{2}}\left(x_{1}, x_{2}\right)$ separately. Our aim is to prove that

$$
\begin{aligned}
S_{1}:= & \sum_{z \in \mathbb{Z}^{d}} \int_{E_{\mathrm{o}}} \int_{E_{z}} \int_{0}^{\infty} \int_{\mathbb{R}^{d}} g\left(d_{B}\left(x_{1}, B(y, r)\right), r\right) g\left(d_{B}\left(x_{2}, B(y, r)\right), r\right) \\
& \times\left|I_{1}\left(\mathbb{R}^{d}, \mathbb{R}^{d}\right)-I_{1}\left(F_{\mathrm{o}}, \mathbb{R}^{d}\right)-I_{1}\left(\mathbb{R}^{d}, F_{z}\right)+I_{1}\left(F_{\mathrm{o}}, F_{z}\right)\right| \mathrm{d} y \mathbb{G}(\mathrm{d} r) \mathrm{d} x_{2} \mathrm{~d} x_{1}
\end{aligned}
$$

and

$$
\begin{aligned}
S_{2}:= & \sum_{z \in \mathbb{Z}^{d}} \int_{E_{\mathrm{o}}} \int_{E_{z}} \int_{0}^{\infty} \int_{0}^{\infty} \int_{\mathbb{R}^{d}} \int_{\mathbb{R}^{d}} g\left(d_{B}\left(x_{1}, B\left(y_{1}, r_{1}\right)\right), r_{1}\right) g\left(d_{B}\left(x_{2}, B\left(y_{2}, r_{2}\right)\right), r_{2}\right) \\
& \times\left|I_{2}\left(\mathbb{R}^{d}, \mathbb{R}^{d}\right)-I_{2}\left(F_{\mathrm{o}}, \mathbb{R}^{d}\right)-I_{2}\left(\mathbb{R}^{d}, F_{z}\right)+I_{2}\left(F_{\mathrm{o}}, F_{z}\right)\right| \mathrm{d} y_{1} \mathrm{~d} y_{2} \mathbb{G}\left(\mathrm{d} r_{1}\right) \mathbb{G}\left(\mathrm{d} r_{2}\right) \mathrm{d} x_{2} \mathrm{~d} x_{1}
\end{aligned}
$$

tend to zero as $m \rightarrow \infty$. Observe that $S_{1}, S_{2}$ depend on $m$ via the dependence of $F_{\mathrm{o}}, F_{z}$ on $m$.

First, we consider $S_{1}$. We rewrite

$$
\begin{aligned}
& I_{1}\left(\mathbb{R}^{d}, \mathbb{R}^{d}\right)-I_{1}\left(F_{\mathrm{o}}, \mathbb{R}^{d}\right)-I_{1}\left(\mathbb{R}^{d}, F_{z}\right)+I_{1}\left(F_{\mathrm{o}}, F_{z}\right) \\
& =\mathbf{1}\left\{y \in F_{\mathrm{o}}^{c} \cap F_{z}^{c}\right\} \bar{F}_{B}^{\mathbb{R}^{d}, \mathbb{R}^{d}}\left(x_{1}, x_{2} ; t_{1}, t_{2}\right)+\mathbf{1}\left\{y \in F_{\mathrm{o}} \cap F_{z}^{c}\right\}\left(\bar{F}_{B}^{\mathbb{R}^{d}, \mathbb{R}^{d}}-\bar{F}_{B}^{F_{\mathrm{o}}, \mathbb{R}^{d}}\right)\left(x_{1}, x_{2} ; t_{1}, t_{2}\right) \\
& +\mathbf{1}\left\{y \in F_{\mathrm{o}}^{c} \cap F_{z}\right\}\left(\bar{F}_{B}^{\mathbb{R}^{d}, \mathbb{R}^{d}}-\bar{F}_{B}^{\mathbb{R}^{d}, F_{z}}\right)\left(x_{1}, x_{2} ; t_{1}, t_{2}\right) \\
& +\mathbf{1}\left\{y \in F_{\mathrm{o}} \cap F_{z}\right\}\left(\bar{F}_{B}^{\mathbb{R}^{d}, \mathbb{R}^{d}}-\bar{F}_{B}^{F_{\mathrm{o}}, \mathbb{R}^{d}}-\bar{F}_{B}^{\mathbb{R}^{d}, F_{z}}+\bar{F}_{B}^{F_{\mathrm{o}}, F_{z}}\right)\left(x_{1}, x_{2} ; t_{1}, t_{2}\right)
\end{aligned}
$$

with $t_{1}=d_{B}\left(x_{1}, B(y, r)\right)$ and $t_{2}=d_{B}\left(x_{2}, B(y, r)\right)$. For notational simplicity, write

$$
\nu_{1}\left(x_{1}, t_{1}\right):=\gamma \mathbb{E}\left|\left(x_{1}+B_{t_{1}, R}\right) \cap F_{\mathrm{o}}^{c}\right|_{d}, \quad \nu_{2}\left(x_{2}, t_{2}\right):=\gamma \mathbb{E}\left|\left(x_{2}+B_{t_{2}, R}\right) \cap F_{z}^{c}\right|_{d}
$$

for $x_{1}, x_{2} \in \mathbb{R}^{d}$ and $t_{1}, t_{2} \in \mathbb{R}^{+}$. We suppress the dependence on $z$ in $\nu_{2}\left(x_{2}, t_{2}\right)$. From (2.3) and the inequality $1-\mathrm{e}^{-a} \leq a$, for $a \geq 0$, we obtain for $x_{1}, x_{2} \in \mathbb{R}^{d}$ and $t_{1}, t_{2} \in \mathbb{R}^{+}$that

$$
\begin{aligned}
& \left|\left(\bar{F}_{B}^{\mathbb{R}^{d}, \mathbb{R}^{d}}-\bar{F}_{B}^{F_{\mathrm{o}}, \mathbb{R}^{d}}\right)\left(x_{1}, x_{2} ; t_{1}, t_{2}\right)\right|=\left(\bar{F}_{B}^{F_{\mathrm{o}}, \mathbb{R}^{d}}-\bar{F}_{B}^{\mathbb{R}^{d}, \mathbb{R}^{d}}\right)\left(x_{1}, x_{2} ; t_{1}, t_{2}\right) \\
& \quad=\bar{F}_{B}^{F_{\mathrm{o}}, \mathbb{R}^{d}}\left(x_{1}, x_{2} ; t_{1}, t_{2}\right)\left(1-\exp \left\{-\gamma \mathbb{E}\left|\left(x_{1}+B_{t_{1}, R}\right) \cap\left(x_{2}+B_{t_{2}, R}\right)^{c} \cap F_{\mathrm{o}}^{c}\right|_{d}\right\}\right) \\
& \quad \leq \nu_{1}\left(x_{1}, t_{1}\right) .
\end{aligned}
$$

Analogously,

$$
\left|\left(\bar{F}_{B}^{\mathbb{R}^{d}, \mathbb{R}^{d}}-\bar{F}_{B}^{\mathbb{R}^{d}, F_{z}}\right)\left(x_{1}, x_{2} ; t_{1}, t_{2}\right)\right| \leq \nu_{2}\left(x_{2}, t_{2}\right) .
$$


Furthermore,

$$
\begin{aligned}
& \left(\bar{F}_{B}^{\mathbb{R}^{d}, \mathbb{R}^{d}}-\bar{F}_{B}^{F_{\mathrm{o}}, \mathbb{R}^{d}}-\bar{F}_{B}^{\mathbb{R}^{d}, F_{z}}+\bar{F}_{B}^{F_{\mathrm{o}}, F_{z}}\right)\left(x_{1}, x_{2} ; t_{1}, t_{2}\right)=\bar{F}_{B}^{F_{\mathrm{o}}, F_{z}}\left(x_{1}, x_{2} ; t_{1}, t_{2}\right) \\
& \quad \times\left(1-\exp \left\{-\gamma \mathbb{E}\left|\left(x_{1}+B_{t_{1}, R}\right) \cap F_{\mathrm{o}}^{c} \cap\left(\left(x_{2}+B_{t_{2}, R}\right)^{c} \cup F_{z}^{c}\right)\right|_{d}\right\}\right) \\
& \quad \times\left(1-\exp \left\{-\gamma \mathbb{E}\left|\left(x_{2}+B_{t_{2}, R}\right) \cap F_{z}^{c} \cap\left(\left(x_{1}+B_{t_{1}, R}\right)^{c} \cup F_{\mathrm{o}}^{c}\right)\right|_{d}\right\}\right) \\
& \quad+\bar{F}_{B}^{\mathbb{R}^{d}, \mathbb{R}^{d}}\left(x_{1}, x_{2} ; t_{1}, t_{2}\right)\left(1-\exp \left\{-\gamma \mathbb{E}\left|\left(x_{1}+B_{t_{1}, R}\right) \cap\left(x_{2}+B_{t_{2}, R}\right) \cap F_{\mathrm{o}}^{c} \cap F_{z}^{c}\right|_{d}\right\}\right)
\end{aligned}
$$

gives

$$
\begin{array}{r}
\left|\left(\bar{F}_{B}^{\mathbb{R}^{d}, \mathbb{R}^{d}}-\bar{F}_{B}^{F_{\mathrm{o}}, \mathbb{R}^{d}}-\bar{F}_{B}^{\mathbb{R}^{d}, F_{z}}+\bar{F}_{B}^{F_{\mathrm{o}}, F_{z}}\right)\left(x_{1}, x_{2} ; t_{1}, t_{2}\right)\right| \\
\leq \nu_{1}\left(x_{1}, t_{1}\right) \nu_{2}\left(x_{2}, t_{2}\right)+\sqrt{\nu_{1}\left(x_{1}, t_{1}\right) \nu_{2}\left(x_{1}, t_{2}\right)}
\end{array}
$$

because by the Cauchy-Schwarz inequality

$$
\mathbb{E}\left|X_{1} \cap X_{2}\right|_{d} \leq \sqrt{\mathbb{E}\left|X_{1}\right|_{d}} \sqrt{\mathbb{E}\left|X_{2}\right|_{d}}
$$

for any random sets $X_{1}$ and $X_{2}$. Combining (3.6), (3.7) and (3.9), we obtain

$$
\begin{aligned}
& \left|I_{1}\left(\mathbb{R}^{d}, \mathbb{R}^{d}\right)-I_{1}\left(F_{\mathrm{o}}, \mathbb{R}^{d}\right)-I_{1}\left(\mathbb{R}^{d}, F_{z}\right)+I_{1}\left(F_{\mathrm{o}}, F_{z}\right)\right| \\
& \leq \mathbf{1}\left\{y \in F_{\mathrm{o}}^{c} \cap F_{z}^{c}\right\}+\mathbf{1}\left\{y \in F_{\mathrm{o}} \cap F_{z}^{c}\right\} \nu_{1}\left(x_{1}, t_{1}\right)+\mathbf{1}\left\{y \in F_{\mathrm{o}}^{c} \cap F_{z}\right\} \nu_{2}\left(x_{2}, t_{2}\right) \\
& \quad+\mathbf{1}\left\{y \in F_{\mathrm{o}} \cap F_{z}\right\}\left[\nu_{1}\left(x_{1}, t_{1}\right) \nu_{2}\left(x_{2}, t_{2}\right)+\sqrt{\nu_{1}\left(x_{1}, t_{1}\right) \nu_{2}\left(x_{2}, t_{2}\right)}\right],
\end{aligned}
$$

where $t_{1}=d_{B}\left(x_{1}, B(y, r)\right)$ and $t_{2}=d_{B}\left(x_{2}, B(y, r)\right)$. If $x_{1} \in E_{\mathrm{o}}$ and $x_{2} \in E_{z}$, then $\nu_{1}\left(x_{1}, t_{1}\right)$ is bounded by $\nu\left(t_{1}\right)$ and $\nu_{2}\left(x_{2}, t_{2}\right)$ is bounded by $\nu\left(t_{2}\right)$, where

$$
\nu(t):=\gamma \mathbb{E}\left|\left(E_{\mathrm{o}} \oplus B_{t, R}\right) \cap F_{\mathrm{o}}^{c}\right|_{d}, \quad t \in \mathbb{R}^{+} .
$$

Let $c_{B}>0$ be such that $B \subset c_{B} B^{d}$. Then

$$
\nu(t) \leq \mathbb{E}\left|B_{t, R+\sqrt{d}}\right|_{d} \leq \kappa_{d} \mathbb{E}\left(c_{B} t+R+\sqrt{d}\right)^{d} \leq c_{1}\left(1+t^{d}\right),
$$

where $c_{1}$ is a finite constant that does not depend on $t$. If $x_{1} \in E_{\mathrm{o}}$ and $y \in F_{\mathrm{o}}^{c}$, then $\left\|x_{1}-y\right\| \geq m$ and $d_{B}\left(x_{1}, B(y, r)\right) \geq(m-r)^{+} / c_{B}$. Similarly, if $x_{2} \in E_{z}$ and $y \in F_{z}^{c}$, then $d_{B}\left(x_{2}, B(y, r)\right) \geq$ $(m-r)^{+} / c_{B}$. Let

$$
\psi(t, r):=\mathbf{1}\left\{t \geq \frac{(m-r)^{+}}{c_{B}}\right\}, \quad t, r \in \mathbb{R}^{+} .
$$

Then, by (3.10) and the substitutions $x_{1}-y \rightarrow x_{1}$ and $x_{2}-y \rightarrow x_{2}$, we get

$$
\begin{aligned}
S_{1} \leq \sum_{z \in \mathbb{Z}^{d}} & \int_{\mathbb{R}^{d}} \int_{\mathbb{R}^{d}} \int_{0}^{\infty} \int_{\mathbb{R}^{d}} g\left(d_{B}\left(x_{1}, r B^{d}\right), r\right) g\left(d_{B}\left(x_{2}, r B^{d}\right), r\right) \mathbf{1}\left\{x_{1}+y \in E_{\mathrm{o}}, x_{2}+y \in E_{z}\right\} \\
\times & {\left[\psi\left(d_{B}\left(x_{1}, r B^{d}\right), r\right) \psi\left(d_{B}\left(x_{2}, r B^{d}\right), r\right)+\psi\left(d_{B}\left(x_{2}, r B^{d}\right), r\right) \nu\left(d_{B}\left(x_{1}, r B^{d}\right)\right)\right.} \\
& +\psi\left(d_{B}\left(x_{1}, r B^{d}\right), r\right) \nu\left(d_{B}\left(x_{2}, r B^{d}\right)\right)+\nu\left(d_{B}\left(x_{1}, r B^{d}\right)\right) \nu\left(d_{B}\left(x_{2}, r B^{d}\right)\right) \\
& \left.+\sqrt{\nu\left(d_{B}\left(x_{1}, r B^{d}\right)\right) \nu\left(d_{B}\left(x_{2}, r B^{d}\right)\right)}\right] \mathrm{d} y \mathbb{G}(\mathrm{d} r) \mathrm{d} x_{2} \mathrm{~d} x_{1}
\end{aligned}
$$


and thus

$$
\begin{aligned}
S_{1} \leq \int_{0}^{\infty} \int_{\mathbb{R}^{d}} \int_{\mathbb{R}^{d}} g\left(d_{B}\left(x_{1}, r B^{d}\right), r\right) g\left(d_{B}\left(x_{2}, r B^{d}\right), r\right) \\
\quad \times v\left(d_{B}\left(x_{1}, r B^{d}\right), d_{B}\left(x_{2}, r B^{d}\right), r\right) \mathrm{d} x_{2} \mathrm{~d} x_{1} \mathbb{G}(\mathrm{d} r),
\end{aligned}
$$

where

$$
v\left(t_{1}, t_{2}, r\right):=\left(\psi\left(t_{1}, r\right)+\nu\left(t_{1}\right)\right)\left(\psi\left(t_{2}, r\right)+\nu\left(t_{2}\right)\right)+\sqrt{\nu\left(t_{1}\right) \nu\left(t_{2}\right)}, \quad t_{1}, t_{2}, r \in \mathbb{R}^{+} .
$$

Now Fubini's theorem, two applications of (2.12) and definition (2.11) of $g$ yield

$$
S_{1} \leq \int_{C} \int_{0}^{\infty} \int_{0}^{\infty} f\left(t_{1}\right) f\left(t_{2}\right) v\left(t_{1}, t_{2}, r\right) \mathrm{d} t_{2} \mathrm{~d} t_{1} \mathbb{G}(\mathrm{d} r) .
$$

Our moment assumption (1.2) ensures that $\mathbb{E}\left|E_{\mathrm{o}} \oplus B_{t, R}\right|_{d}<\infty$ and therefore $\nu(t) \rightarrow 0$ as $m \rightarrow \infty$ by Lebesgue's dominated convergence theorem. By (3.11) and (3.1), another application of Lebesgue's dominated convergence theorem shows that $S_{1} \rightarrow 0$ as $m \rightarrow \infty$.

Next, we proceed with $S_{2}$. From (2.4) and the inequality $1-\mathrm{e}^{-a} \leq a$, for $a \geq 0$, we obtain for $x_{1}, x_{2} \in \mathbb{R}^{d}$ and $t_{1}, t_{2} \in \mathbb{R}^{+}$that

$$
\begin{aligned}
\bar{F}_{B}^{\mathbb{R}^{d}, \mathbb{R}^{d}}\left(x_{1}, x_{2} ; t_{1}, t_{2}\right)-\bar{F}_{B}^{\mathbb{R}^{d}}\left(x_{1} ; t_{1}\right) \bar{F}_{B}^{\mathbb{R}^{d}}\left(x_{2} ; t_{2}\right) \\
\quad=\bar{F}_{B}^{\mathbb{R}^{d}, \mathbb{R}^{d}}\left(x_{1}, x_{2} ; t_{1}, t_{2}\right)\left(1-\exp \left\{-\gamma \mathbb{E} \kappa_{B}\left(x_{2}-x_{1} ; t_{1}, t_{2}, R\right)\right\}\right) \\
\quad \leq \gamma \mathbb{E} \kappa_{B}\left(x_{2}-x_{1} ; t_{1}, t_{2}, R\right),
\end{aligned}
$$

where $\kappa_{B}$ is defined in $(2.5$, and

$$
\begin{aligned}
& \left(\bar{F}_{B}^{\mathbb{R}^{d}, \mathbb{R}^{d}}-\bar{F}_{B}^{F_{\mathrm{o}}, \mathbb{R}^{d}}\right)\left(x_{1}, x_{2} ; t_{1}, t_{2}\right)-\left(\bar{F}_{B}^{\mathbb{R}^{d}}-\bar{F}_{B}^{F_{\mathrm{o}}}\right)\left(x_{1} ; t_{1}\right) \bar{F}_{B}^{\mathbb{R}^{d}}\left(x_{2} ; t_{2}\right) \\
& =-\bar{F}_{B}^{F_{\mathrm{o}}, \mathbb{R}^{d}}\left(x_{1}, x_{2} ; t_{1}, t_{2}\right)\left(1-\exp \left\{-\gamma \mathbb{E}\left|\left(x_{1}+B_{t_{1}, R}\right) \cap\left(x_{2}+B_{t_{2}, R}\right) \cap F_{\mathrm{o}}\right|_{d}\right\}\right) \\
& +\bar{F}_{B}^{\mathbb{R}^{d}, \mathbb{R}^{d}}\left(x_{1}, x_{2} ; t_{1}, t_{2}\right)\left(1-\exp \left\{-\gamma \mathbb{E}\left|\left(x_{1}+B_{t_{1}, R}\right) \cap\left(x_{2}+B_{t_{2}, R}\right)\right|_{d}\right\}\right) .
\end{aligned}
$$

Therefore,

$$
\begin{aligned}
& \left|\left(\bar{F}_{B}^{\mathbb{R}^{d}, \mathbb{R}^{d}}-\bar{F}_{B}^{F_{\mathrm{o}}, \mathbb{R}^{d}}\right)\left(x_{1}, x_{2} ; t_{1}, t_{2}\right)-\left(\bar{F}_{B}^{\mathbb{R}^{d}}-\bar{F}_{B}^{F_{\mathrm{o}}}\right)\left(x_{1} ; t_{1}\right) \bar{F}_{B}^{\mathbb{R}^{d}}\left(x_{2} ; t_{2}\right)\right| \\
& \quad \leq \gamma \mathbb{E} \kappa_{B}\left(x_{2}-x_{1} ; t_{1}, t_{2}, R\right) .
\end{aligned}
$$

Analogously,

$$
\begin{aligned}
& \left|\left(\bar{F}_{B}^{\mathbb{R}^{d}, \mathbb{R}^{d}}-\bar{F}_{B}^{\mathbb{R}^{d}, F_{z}}\right)\left(x_{1}, x_{2} ; t_{1}, t_{2}\right)-\bar{F}_{B}^{\mathbb{R}^{d}}\left(x_{1} ; t_{1}\right)\left(\bar{F}_{B}^{\mathbb{R}^{d}}-\bar{F}_{B}^{F_{z}}\right)\left(x_{2} ; t_{2}\right)\right| \\
& \quad \leq \gamma \mathbb{E} \kappa_{B}\left(x_{2}-x_{1} ; t_{1}, t_{2}, R\right) .
\end{aligned}
$$


Finally, using (3.8) we get

$$
\begin{aligned}
& \left(\bar{F}_{B}^{\mathbb{R}^{d}, \mathbb{R}^{d}}-\bar{F}_{B}^{F_{\mathrm{o}}, \mathbb{R}^{d}}-\bar{F}_{B}^{\mathbb{R}^{d}, F_{z}}+\bar{F}_{B}^{F_{\mathrm{o}}, F_{z}}\right)\left(x_{1}, x_{2} ; t_{1}, t_{2}\right) \\
& -\left(\bar{F}_{B}^{\mathbb{R}^{d}}-\bar{F}_{B}^{F_{\mathrm{o}}}\right)\left(x_{1} ; t_{1}\right)\left(\bar{F}_{B}^{\mathbb{R}^{d}}-\bar{F}_{B}^{F_{z}}\right)\left(x_{2} ; t_{2}\right) \\
& =\bar{F}_{B}^{F_{\mathrm{o}}, F_{z}}\left(x_{1}, x_{2} ; t_{1}, t_{2}\right)\left(1-\exp \left\{-\gamma \mathbb{E}\left|\left(x_{1}+B_{t_{1}, R}\right) \cap F_{\mathrm{o}}^{c} \cap\left(\left(x_{2}+B_{t_{2}, R}\right)^{c} \cup F_{z}^{c}\right)\right|_{d}\right\}\right) \\
& \times\left(1-\exp \left\{-\gamma \mathbb{E}\left|\left(x_{2}+B_{t_{2}, R}\right) \cap F_{z}^{c} \cap\left(\left(x_{1}+B_{t_{1}, R}\right)^{c} \cup F_{\mathrm{o}}^{c}\right)\right|_{d}\right\}\right) \\
& +\bar{F}_{B}^{\mathbb{R}^{d}, \mathbb{R}^{d}}\left(x_{1}, x_{2} ; t_{1}, t_{2}\right)\left(1-\exp \left\{-\gamma \mathbb{E}\left|\left(x_{1}+B_{t_{1}, R}\right) \cap\left(x_{2}+B_{t_{2}, R}\right) \cap F_{\mathrm{o}}^{c} \cap F_{z}^{c}\right|_{d}\right\}\right) \\
& -\bar{F}_{B}^{F_{\mathrm{o}}}\left(x_{1} ; t_{1}\right) \bar{F}_{B}^{F_{z}}\left(x_{2} ; t_{2}\right)\left(1-\exp \left\{-\nu_{1}\left(x_{1}, t_{1}\right)\right\}\right)\left(1-\exp \left\{-\nu_{2}\left(x_{2}, t_{2}\right)\right\}\right) \\
& =\bar{F}_{B}^{F_{\mathrm{o}}, F_{z}}\left(x_{1}, x_{2} ; t_{1}, t_{2}\right)\left(1-\exp \left\{-\gamma \mathbb{E}\left|\left(x_{1}+B_{t_{1}, R}\right) \cap F_{\mathrm{o}}^{c} \cap\left(\left(x_{2}+B_{t_{2}, R}\right)^{c} \cup F_{z}^{c}\right)\right|_{d}\right\}\right) \\
& \times\left(1-\exp \left\{-\gamma \mathbb{E}\left|\left(x_{2}+B_{t_{2}, R}\right) \cap F_{z}^{c} \cap\left(\left(x_{1}+B_{t_{1}, R}\right)^{c} \cup F_{\mathrm{o}}^{c}\right)\right|_{d}\right\}\right) \\
& \times\left(1-\exp \left\{-\gamma \mathbb{E}\left|\left(x_{1}+B_{t_{1}, R}\right) \cap\left(x_{2}+B_{t_{2}, R}\right) \cap F_{\mathrm{o}} \cap F_{z}\right|_{d}\right\}\right) \\
& +\bar{F}_{B}^{\mathbb{R}^{d}, \mathbb{R}^{d}}\left(x_{1}, x_{2} ; t_{1}, t_{2}\right)\left(1-\exp \left\{-\gamma \mathbb{E}\left|\left(x_{1}+B_{t_{1}, R}\right) \cap\left(x_{2}+B_{t_{2}, R}\right) \cap F_{\mathrm{o}}^{c} \cap F_{z}^{c}\right|_{d}\right\}\right) \\
& -\bar{F}_{B}^{F_{\mathrm{o}}}\left(x_{1} ; t_{1}\right) \bar{F}_{B}^{F_{z}}\left(x_{2} ; t_{2}\right)\left[\exp \left\{-\gamma \mathbb{E}\left|\left(x_{2}+B_{t_{2}, R}\right) \cap F_{z}^{c} \cap\left(\left(x_{1}+B_{t_{1}, R}\right)^{c} \cup F_{\mathrm{o}}^{c}\right)\right|_{d}\right\}\right. \\
& \times\left(1-\exp \left\{-\gamma \mathbb{E}\left|\left(x_{1}+B_{t_{1}, R}\right) \cap\left(x_{2}+B_{t_{2}, R}\right) \cap F_{\mathrm{o}} \cap F_{z}^{c}\right|_{d}\right\}\right)\left(1-\exp \left\{-\nu_{1}\left(x_{1}, t_{1}\right)\right\}\right) \\
& +\exp \left\{-\gamma \mathbb{E}\left|\left(x_{1}+B_{t_{1}, R}\right) \cap F_{\mathrm{o}}^{c} \cap\left(\left(x_{2}+B_{t_{2}, R}\right)^{c} \cup F_{z}^{c}\right)\right|_{d}\right\} \\
& \times\left(1-\exp \left\{-\gamma \mathbb{E}\left|\left(x_{1}+B_{t_{1}, R}\right) \cap\left(x_{2}+B_{t_{2}, R}\right) \cap F_{\mathrm{o}}^{c} \cap F_{z}\right|_{d}\right\}\right) \\
& \left.\times\left(1-\exp \left\{-\gamma \mathbb{E}\left|\left(x_{2}+B_{t_{2}, R}\right) \cap F_{z}^{c} \cap\left(\left(x_{1}+B_{t_{1}, R}\right)^{c} \cup F_{\mathrm{o}}^{c}\right)\right|_{d}\right\}\right)\right],
\end{aligned}
$$

which leads to

$$
\begin{aligned}
& \mid\left(\bar{F}_{B}^{\mathbb{R}^{d}, \mathbb{R}^{d}}-\bar{F}_{B}^{F_{\mathrm{o}}, \mathbb{R}^{d}}-\bar{F}_{B}^{\mathbb{R}^{d}, F_{z}}+\bar{F}_{B}^{F_{\mathrm{o}}, F_{z}}\right)\left(x_{1}, x_{2} ; t_{1}, t_{2}\right) \\
& \quad-\left(\bar{F}_{B}^{\mathbb{R}^{d}}-\bar{F}_{B}^{F_{\mathrm{o}}}\right)\left(x_{1} ; t_{1}\right)\left(\bar{F}_{B}^{\mathbb{R}^{d}}-\bar{F}_{B}^{F_{z}}\right)\left(x_{2} ; t_{2}\right) \mid \\
& \quad \leq \nu_{1}\left(x_{1}, t_{1}\right) \nu_{2}\left(x_{2}, t_{2}\right) \gamma \mathbb{E} \kappa_{B}\left(x_{2}-x_{1} ; t_{1}, t_{2}, R\right) \\
& \quad+\gamma \mathbb{E}\left|\left(x_{1}+B_{t_{1}, R}\right) \cap\left(x_{2}+B_{t_{2}, R}\right) \cap F_{\mathrm{o}}^{c} \cap F_{z}^{c}\right|_{d} \\
& \quad+\nu_{1}\left(x_{1}, t_{1}\right) \gamma \mathbb{E} \kappa_{B}\left(x_{2}-x_{1} ; t_{1}, t_{2}, R\right)+\nu_{2}\left(x_{2}, t_{2}\right) \gamma \mathbb{E} \kappa_{B}\left(x_{2}-x_{1} ; t_{1}, t_{2}, R\right) .
\end{aligned}
$$

In the following, we use (3.12) - (3.15) with $t_{1}=d_{B}\left(x_{1}, B\left(y_{1}, r_{1}\right)\right), t_{2}=d_{B}\left(x_{2}, B\left(y_{2}, r_{2}\right)\right)$. Moreover, we define

$$
\begin{aligned}
& \chi_{1}:=\mathbf{1}\left\{d_{B}\left(x_{1}, B\left(y_{2}, r_{2}\right)\right) \leq t_{1}\right\}=\mathbf{1}\left\{x_{1} \in y_{2}+B_{t_{1}, r_{2}}\right\}, \\
& \chi_{2}:=\mathbf{1}\left\{d_{B}\left(x_{2}, B\left(y_{1}, r_{1}\right)\right) \leq t_{2}\right\}=\mathbf{1}\left\{x_{2} \in y_{1}+B_{t_{2}, r_{1}}\right\},
\end{aligned}
$$


and

$$
\tilde{\kappa}_{B}\left(x_{1}, x_{2} ; t_{1}, t_{2}, R\right):=\left|\left(x_{1}+B_{t_{1}, R}\right) \cap\left(x_{2}+B_{t_{2}, R}\right) \cap F_{\mathrm{o}}^{c}\right|_{d} .
$$

Next, we fix $x_{1} \in E_{\mathrm{o}}$ and $x_{2} \in E_{z}$, for the moment, and distinguish several cases.

1. If $y_{1} \in F_{\mathrm{o}}^{c}$ and $y_{2} \in F_{z}^{c}$, then $t_{1} \geq\left(m-r_{1}\right)^{+} / c_{B}, t_{2} \geq\left(m-r_{2}\right)^{+} / c_{B}$ and using (3.12) we get

$$
\begin{aligned}
& \left|I_{2}\left(\mathbb{R}^{d}, \mathbb{R}^{d}\right)-I_{2}\left(F_{\mathrm{o}}, \mathbb{R}^{d}\right)-I_{2}\left(\mathbb{R}^{d}, F_{z}\right)+I_{2}\left(F_{\mathrm{o}}, F_{z}\right)\right|=\left|I_{2}\left(\mathbb{R}^{d}, \mathbb{R}^{d}\right)\right| \\
& \quad=\left|\left(1-\chi_{1}\right)\left(1-\chi_{2}\right) \bar{F}_{B}^{\mathbb{R}^{d}, \mathbb{R}^{d}}\left(x_{1}, x_{2} ; t_{1}, t_{2}\right)-\bar{F}_{B}\left(t_{1}\right) \bar{F}_{B}\left(t_{2}\right)\right| \\
& \quad \leq \gamma \mathbb{E} \kappa_{B}\left(x_{2}-x_{1} ; t_{1}, t_{2}, R\right)+\chi_{1}+\chi_{2} .
\end{aligned}
$$

2. If $y_{1} \in F_{\mathrm{o}}$ and $y_{2} \in F_{z}^{c} \cap F_{\mathrm{o}}$, then $t_{2} \geq\left(m-r_{2}\right)^{+} / c_{B}$ and using (3.13) we get

$$
\begin{aligned}
& \left|I_{2}\left(\mathbb{R}^{d}, \mathbb{R}^{d}\right)-I_{2}\left(F_{\mathrm{o}}, \mathbb{R}^{d}\right)-I_{2}\left(\mathbb{R}^{d}, F_{z}\right)+I_{2}\left(F_{\mathrm{o}}, F_{z}\right)\right|=\left|I_{2}\left(\mathbb{R}^{d}, \mathbb{R}^{d}\right)-I_{2}\left(F_{\mathrm{o}}, \mathbb{R}^{d}\right)\right| \\
& \quad=\left|\left(1-\chi_{1}\right)\left(1-\chi_{2}\right)\left(\bar{F}_{B}^{\mathbb{R}^{d}, \mathbb{R}^{d}}-\bar{F}_{B}^{F_{\mathrm{o}}, \mathbb{R}^{d}}\right)\left(x_{1}, x_{2} ; t_{1}, t_{2}\right)-\left(\bar{F}_{B}^{\mathbb{R}^{d}}-\bar{F}_{B}^{F_{\mathrm{o}}}\right)\left(x_{1} ; t_{1}\right) \bar{F}_{B}\left(t_{2}\right)\right| \\
& \quad \leq \gamma \mathbb{E} \kappa_{B}\left(x_{2}-x_{1} ; s_{1}, s_{2}, R\right)+\chi_{1}+\chi_{2} .
\end{aligned}
$$

3. If $y_{1} \in F_{\mathrm{o}}$ and $y_{2} \in F_{z}^{c} \cap F_{\mathrm{o}}^{c}$, then $t_{2} \geq\left(m-r_{2}\right)^{+} / c_{B}$ and using (3.13) we get

$$
\begin{aligned}
& \left|I_{2}\left(\mathbb{R}^{d}, \mathbb{R}^{d}\right)-I_{2}\left(F_{\mathrm{o}}, \mathbb{R}^{d}\right)-I_{2}\left(\mathbb{R}^{d}, F_{z}\right)+I_{2}\left(F_{\mathrm{o}}, F_{z}\right)\right|=\left|I_{2}\left(\mathbb{R}^{d}, \mathbb{R}^{d}\right)-I_{2}\left(F_{\mathrm{o}}, \mathbb{R}^{d}\right)\right| \\
& \quad=\left|\left(1-\chi_{2}\right)\left(\left(1-\chi_{1}\right) \bar{F}_{B}^{\mathbb{R}^{d}, \mathbb{R}^{d}}-\bar{F}_{B}^{F_{\mathrm{o}}, \mathbb{R}^{d}}\right)\left(x_{1}, x_{2} ; t_{1}, t_{2}\right)-\left(\bar{F}_{B}^{\mathbb{R}^{d}}-\bar{F}_{B}^{F_{\mathrm{o}}}\right)\left(x_{1} ; t_{1}\right) \bar{F}_{B}\left(t_{2}\right)\right| \\
& \quad \leq \gamma \mathbb{E} \kappa_{B}\left(x_{2}-x_{1} ; s_{1}, s_{2}, R\right)+\chi_{1}+\chi_{2} .
\end{aligned}
$$

4. If $y_{1} \in F_{o}^{c} \cap F_{z}$ and $y_{2} \in F_{z}$, then $t_{1} \geq\left(m-r_{1}\right)^{+} / c_{B}$ and using (3.14) we get

$$
\begin{aligned}
& \left|I_{2}\left(\mathbb{R}^{d}, \mathbb{R}^{d}\right)-I_{2}\left(F_{\mathrm{o}}, \mathbb{R}^{d}\right)-I_{2}\left(\mathbb{R}^{d}, F_{z}\right)+I_{2}\left(F_{\mathrm{o}}, F_{z}\right)\right|=\left|I_{2}\left(\mathbb{R}^{d}, \mathbb{R}^{d}\right)-I_{2}\left(\mathbb{R}^{d}, F_{z}\right)\right| \\
& \quad=\left|\left(1-\chi_{1}\right)\left(1-\chi_{2}\right)\left(\bar{F}_{B}^{\mathbb{R}^{d}, \mathbb{R}^{d}}-\bar{F}_{B}^{\mathbb{R}^{d}, F_{z}}\right)\left(x_{1}, x_{2} ; t_{1}, t_{2}\right)-\bar{F}_{B}\left(t_{1}\right)\left(\bar{F}_{B}^{\mathbb{R}^{d}}-\bar{F}_{B}^{F_{z}}\right)\left(x_{2} ; t_{2}\right)\right| \\
& \quad \leq \gamma \mathbb{E} \kappa_{B}\left(x_{2}-x_{1} ; t_{1}, t_{2}, R\right)+\chi_{1}+\chi_{2} .
\end{aligned}
$$

5. If $y_{1} \in F_{\mathrm{o}}^{c} \cap F_{z}^{c}$ and $y_{2} \in F_{z}$, then $t_{1} \geq\left(m-r_{1}\right)^{+} / c_{B}$ and using (3.14) we get

$$
\begin{aligned}
& \left|I_{2}\left(\mathbb{R}^{d}, \mathbb{R}^{d}\right)-I_{2}\left(F_{\mathrm{o}}, \mathbb{R}^{d}\right)-I_{2}\left(\mathbb{R}^{d}, F_{z}\right)+I_{2}\left(F_{\mathrm{o}}, F_{z}\right)\right|=\left|I_{2}\left(\mathbb{R}^{d}, \mathbb{R}^{d}\right)-I_{2}\left(\mathbb{R}^{d}, F_{z}\right)\right| \\
& \quad=\left|\left(1-\chi_{1}\right)\left(\left(1-\chi_{2}\right) \bar{F}_{B}^{\mathbb{R}^{d}, \mathbb{R}^{d}}-\bar{F}_{B}^{\mathbb{R}^{d}, F_{z}}\right)\left(x_{1}, x_{2} ; t_{1}, t_{2}\right)-\bar{F}_{B}\left(t_{1}\right)\left(\bar{F}_{B}^{\mathbb{R}^{d}}-\bar{F}_{B}^{F_{z}}\right)\left(x_{2} ; t_{2}\right)\right| \\
& \quad \leq \gamma \mathbb{E} \kappa_{B}\left(x_{2}-x_{1} ; t_{1}, t_{2}, R\right)+\chi_{1}+\chi_{2} .
\end{aligned}
$$


6. If $y_{1} \in F_{\mathrm{o}} \cap F_{z}$ and $y_{2} \in F_{z} \cap F_{\mathrm{o}}$, then by (3.9) and (3.15),

$$
\begin{aligned}
&\left|I_{2}\left(\mathbb{R}^{d}, \mathbb{R}^{d}\right)-I_{2}\left(F_{\mathrm{o}}, \mathbb{R}^{d}\right)-I_{2}\left(\mathbb{R}^{d}, F_{z}\right)+I_{2}\left(F_{\mathrm{o}}, F_{z}\right)\right| \\
&=\mid \mid\left(1-\chi_{1}\right)\left(1-\chi_{2}\right)\left(\bar{F}_{B}^{\mathbb{R}^{d}, \mathbb{R}^{d}}-\bar{F}_{B}^{F_{\mathrm{o}}, \mathbb{R}^{d}}-\bar{F}_{B}^{\mathbb{R}^{d}, F_{z}}+\bar{F}_{B}^{F_{\mathrm{o}}, F_{z}}\right)\left(x_{1}, x_{2} ; t_{1}, t_{2}\right) \\
&-\left(\bar{F}_{B}^{\mathbb{R}^{d}}-\bar{F}_{B}^{F_{\mathrm{o}}}\right)\left(x_{1} ; t_{1}\right)\left(\bar{F}_{B}^{\mathbb{R}^{d}}-\bar{F}_{B}^{F_{z}}\right)\left(x_{2} ; t_{2}\right) \mid \\
& \leq\left(\nu_{1}\left(x_{1}, t_{1}\right) \nu_{2}\left(x_{2}, t_{2}\right)+\nu_{1}\left(x_{1}, t_{1}\right)+\nu_{2}\left(x_{2}, t_{2}\right)\right) \gamma \mathbb{E} \kappa_{B}\left(x_{2}-x_{1} ; t_{1}, t_{2}, R\right) \\
&+\gamma \mathbb{E} \tilde{\kappa}_{B}\left(x_{1}, x_{2} ; t_{1}, t_{2}, R\right)+\left(\chi_{1}+\chi_{2}\right)\left(\nu_{1}\left(x_{1}, t_{1}\right) \nu_{2}\left(x_{2}, t_{2}\right)+\sqrt{\nu_{1}\left(x_{1}, t_{1}\right) \nu_{2}\left(x_{2}, t_{2}\right)}\right) .
\end{aligned}
$$

7. If $y_{1} \in F_{\mathrm{o}} \cap F_{z}^{c}$ and $y_{2} \in F_{z} \cap F_{\mathrm{o}}$, then by (3.6), (3.9) and (3.15),

$$
\begin{aligned}
& \left|I_{2}\left(\mathbb{R}^{d}, \mathbb{R}^{d}\right)-I_{2}\left(F_{\mathrm{o}}, \mathbb{R}^{d}\right)-I_{2}\left(\mathbb{R}^{d}, F_{z}\right)+I_{2}\left(F_{\mathrm{o}}, F_{z}\right)\right|=\mid\left(1-\chi_{1}\right)\left(1-\chi_{2}\right) \\
& \quad \times\left(\bar{F}_{B}^{\mathbb{R}^{d}, \mathbb{R}^{d}}-\bar{F}_{B}^{F_{\mathrm{o}}, \mathbb{R}^{d}}\right)\left(x_{1}, x_{2} ; t_{1}, t_{2}\right)-\left(1-\chi_{1}\right)\left(\bar{F}_{B}^{\mathbb{R}^{d}, F_{z}}-\bar{F}_{B}^{F_{\mathrm{o}}, F_{z}}\right)\left(x_{1}, x_{2} ; t_{1}, t_{2}\right) \\
& \quad-\left(\bar{F}_{B}^{\mathbb{R}^{d}}-\bar{F}_{B}^{F_{\mathrm{o}}}\right)\left(x_{1} ; t_{1}\right)\left(\bar{F}_{B}^{\mathbb{R}^{d}}-\bar{F}_{B}^{F_{z}}\right)\left(x_{2} ; t_{2}\right) \mid \\
& \leq\left(\nu_{1}\left(x_{1}, t_{1}\right) \nu_{2}\left(x_{2}, t_{2}\right)+\nu_{1}\left(x_{1}, t_{1}\right)+\nu_{2}\left(x_{2}, t_{2}\right)\right) \gamma \mathbb{E} \kappa_{B}\left(x_{2}-x_{1} ; t_{1}, t_{2}, R\right) \\
& \quad+\gamma \mathbb{E} \tilde{\kappa}_{B}\left(x_{1}, x_{2} ; t_{1}, t_{2}, R\right)+\chi_{1}\left(\nu_{1}\left(x_{1}, t_{1}\right) \nu_{2}\left(x_{2}, t_{2}\right)+\sqrt{\nu_{1}\left(x_{1}, t_{1}\right) \nu_{2}\left(x_{2}, t_{2}\right)}\right) \\
& \quad+\chi_{2} \nu_{1}\left(x_{1}, t_{1}\right) .
\end{aligned}
$$

8. If $y_{1} \in F_{\mathrm{o}} \cap F_{z}$ and $y_{2} \in F_{z} \cap F_{\mathrm{o}}^{c}$, then by (3.7), (3.9) and (3.15),

$$
\begin{aligned}
& \left|I_{2}\left(\mathbb{R}^{d}, \mathbb{R}^{d}\right)-I_{2}\left(F_{\mathrm{o}}, \mathbb{R}^{d}\right)-I_{2}\left(\mathbb{R}^{d}, F_{z}\right)+I_{2}\left(F_{\mathrm{o}}, F_{z}\right)\right|=\mid\left(1-\chi_{1}\right)\left(1-\chi_{2}\right) \\
& \quad \times\left(\bar{F}_{B}^{\mathbb{R}^{d}, \mathbb{R}^{d}}-\bar{F}_{B}^{\mathbb{R}^{d}, F_{z}}\right)\left(x_{1}, x_{2} ; t_{1}, t_{2}\right)-\left(1-\chi_{2}\right)\left(\bar{F}_{B}^{F_{\mathrm{o}}, \mathbb{R}^{d}}-\bar{F}_{B}^{F_{\mathrm{o}}, F_{z}}\right)\left(x_{1}, x_{2} ; t_{1}, t_{2}\right) \\
& \quad-\left(\bar{F}_{B}^{\mathbb{R}^{d}}-\bar{F}_{B}^{F_{\mathrm{o}}}\right)\left(x_{1} ; t_{1}\right)\left(\bar{F}_{B}^{\mathbb{R}^{d}}-\bar{F}_{B}^{F_{z}}\right)\left(x_{2} ; t_{2}\right) \mid \\
& \leq\left(\nu_{1}\left(x_{1}, t_{1}\right) \nu_{2}\left(x_{2}, t_{2}\right)+\nu_{1}\left(x_{1}, t_{1}\right)+\nu_{2}\left(x_{2}, t_{2}\right)\right) \gamma \mathbb{E} \kappa_{B}\left(x_{2}-x_{1} ; t_{1}, t_{2}, R\right) \\
& \quad+\gamma \mathbb{E} \tilde{\kappa}_{B}\left(x_{1}, x_{2} ; t_{1}, t_{2}, R\right)+\chi_{1} \nu_{2}\left(x_{2}, t_{2}\right) \\
& \quad+\chi_{2}\left(\nu_{1}\left(x_{1}, t_{1}\right) \nu_{2}\left(x_{2}, t_{2}\right)+\sqrt{\nu_{1}\left(x_{1}, t_{1}\right) \nu_{2}\left(x_{2}, t_{2}\right)}\right) .
\end{aligned}
$$

9. If $y_{1} \in F_{\mathrm{o}} \cap F_{z}^{c}$ and $y_{2} \in F_{z} \cap F_{\mathrm{o}}^{c}$, then $\chi_{1}=1$ implies $t_{1} \geq\left(m-r_{2}\right)^{+} / c_{B}$ and $\chi_{2}=1$ implies 
$t_{2} \geq\left(m-r_{1}\right)^{+} / c_{B}$, and by (3.15) we have

$$
\begin{aligned}
& \left|I_{2}\left(\mathbb{R}^{d}, \mathbb{R}^{d}\right)-I_{2}\left(F_{\mathrm{o}}, \mathbb{R}^{d}\right)-I_{2}\left(\mathbb{R}^{d}, F_{z}\right)+I_{2}\left(F_{\mathrm{o}}, F_{z}\right)\right|=\mid\left(\left(1-\chi_{1}\right)\left(1-\chi_{2}\right) \bar{F}_{B}^{\mathbb{R}^{d}, \mathbb{R}^{d}}\right. \\
& \left.\quad-\left(1-\chi_{2}\right) \bar{F}_{B}^{F_{\mathrm{o}}, \mathbb{R}^{d}}-\left(1-\chi_{1}\right) \bar{F}_{B}^{\mathbb{R}^{d}, F_{z}}+\bar{F}_{B}^{F_{\mathrm{o}}, F_{z}}\right)\left(x_{1}, x_{2} ; t_{1}, t_{2}\right) \\
& \quad-\left(\bar{F}_{B}^{\mathbb{R}^{d}}-\bar{F}_{B}^{F_{\mathrm{o}}}\right)\left(x_{1} ; t_{1}\right)\left(\bar{F}_{B}^{\mathbb{R}^{d}}-\bar{F}_{B}^{F_{z}}\right)\left(x_{2} ; t_{2}\right) \mid \\
& \leq\left(\nu_{1}\left(x_{1}, t_{1}\right) \nu_{2}\left(x_{2}, t_{2}\right)+\nu_{1}\left(x_{1}, t_{1}\right)+\nu_{2}\left(x_{2}, t_{2}\right)\right) \gamma \mathbb{E} \kappa_{B}\left(x_{2}-x_{1} ; t_{1}, t_{2}, R\right) \\
& \quad+\gamma \mathbb{E} \tilde{\kappa}_{B}\left(x_{1}, x_{2} ; t_{1}, t_{2}, R\right)+\chi_{1}+\chi_{2} .
\end{aligned}
$$

Altogether this gives

$$
\left|I_{2}\left(\mathbb{R}^{d}, \mathbb{R}^{d}\right)-I_{2}\left(F_{\mathrm{o}}, \mathbb{R}^{d}\right)-I_{2}\left(\mathbb{R}^{d}, F_{z}\right)+I_{2}\left(F_{\mathrm{o}}, F_{z}\right)\right| \leq I_{2}^{*}\left(x_{1}, x_{2}, y_{1}, y_{2}, r_{1}, r_{2}\right),
$$

where

$$
\begin{aligned}
I_{2}^{*} & \left.x_{1}, x_{2}, y_{1}, y_{2}, r_{1}, r_{2}\right):=\left(\psi\left(t_{1}, r_{1}\right) \psi\left(t_{2}, r_{2}\right)+2 \psi\left(t_{2}, r_{2}\right)+2 \psi\left(t_{1}, r_{1}\right)\right) \\
& \quad \times\left(\gamma \mathbb{E} \kappa_{B}\left(x_{2}-x_{1} ; t_{1}, t_{2}, R\right)+\chi_{1}+\chi_{2}\right) \\
+ & 4\left(\nu\left(t_{1}\right) \nu\left(t_{2}\right)+\nu\left(t_{1}\right)+\nu\left(t_{2}\right)\right) \gamma \mathbb{E} \kappa_{B}\left(x_{2}-x_{1} ; t_{1}, t_{2}, R\right)+4 \gamma \mathbb{E} \tilde{\kappa}_{B}\left(x_{1}, x_{2} ; t_{1}, t_{2}, R\right) \\
+ & 2\left(\chi_{1}+\chi_{2}\right)\left(\nu\left(t_{1}\right) \nu\left(t_{2}\right)+\sqrt{\nu\left(t_{1}\right) \nu\left(t_{2}\right)}\right) \\
& +\chi_{2} \nu\left(t_{1}\right)+\chi_{1} \nu\left(t_{2}\right)+\chi_{1} \psi\left(t_{1}, r_{2}\right)+\chi_{2} \psi\left(t_{2}, r_{1}\right)
\end{aligned}
$$

does not depend on $z$. We recall the dependence of $\chi_{1}, \chi_{2}$ on $x_{1}, x_{2}, y_{1}, y_{2}$ and then carry out the substitutions $x_{1}-y_{1} \rightarrow x_{1}$ and $x_{2}-y_{2} \rightarrow x_{2}$ to get

$$
\begin{aligned}
S_{2} \leq & \sum_{z \in \mathbb{Z}^{d}} \int_{\mathbb{R}^{d}} \int_{\mathbb{R}^{d}} \int_{0}^{\infty} \int_{0}^{\infty} \int_{\mathbb{R}^{d}} \int_{\mathbb{R}^{d}} g\left(d_{B}\left(x_{1}, r_{1} B^{d}\right), r_{1}\right) g\left(d_{B}\left(x_{2}, r_{2} B^{d}\right), r_{2}\right) \mathbf{1}\left\{x_{1}+y_{1} \in E_{\mathrm{o}}\right\} \\
& \times \mathbf{1}\left\{x_{2}+y_{2} \in E_{z}\right\} I_{2}^{*}\left(x_{1}+y_{1}, x_{2}+y_{2}, y_{1}, y_{2}, r_{1}, r_{2}\right) \mathrm{d} y_{1} \mathrm{~d} y_{2} \mathbb{G}\left(\mathrm{d} r_{1}\right) \mathbb{G}\left(\mathrm{d} r_{2}\right) \mathrm{d} x_{2} \mathrm{~d} x_{1} \\
= & \int_{\mathbb{R}^{d}} \int_{\mathbb{R}^{d}} \int_{0}^{\infty} \int_{0}^{\infty} \int_{\mathbb{R}^{d}} \int_{\mathbb{R}^{d}} g\left(d_{B}\left(x_{1}, r_{1} B^{d}\right), r_{1}\right) g\left(d_{B}\left(x_{2}, r_{2} B^{d}\right), r_{2}\right) \mathbf{1}\left\{x_{1}+y_{1} \in E_{\mathrm{o}}\right\} \\
& \times I_{2}^{*}\left(x_{1}+y_{1}, x_{2}+y_{2}, y_{1}, y_{2}, r_{1}, r_{2}\right) \mathrm{d} y_{1} \mathrm{~d} y_{2} \mathbb{G}\left(\mathrm{d} r_{1}\right) \mathbb{G}\left(\mathrm{d} r_{2}\right) \mathrm{d} x_{2} \mathrm{~d} x_{1} .
\end{aligned}
$$

To the inner integrals we apply the relations

$$
\begin{aligned}
& \int_{\mathbb{R}^{d}} \int_{\mathbb{R}^{d}} \mathbf{1}\left\{x_{1}+y_{1} \in E_{\mathrm{o}}\right\} \chi_{1} \mathrm{~d} y_{2} \mathrm{~d} y_{1}=\int_{E_{\mathrm{o}}-x_{1}} \int_{\mathbb{R}^{d}} \mathbf{1}\left\{x_{1}+y_{1}-y_{2} \in B_{t_{1}, r_{2}}\right\} \mathrm{d} y_{2} \mathrm{~d} y_{1}=\left|B_{t_{1}, r_{2}}\right|_{d}, \\
& \int_{\mathbb{R}^{d}} \int_{\mathbb{R}^{d}} \mathbf{1}\left\{x_{1}+y_{1} \in E_{\mathrm{o}}\right\} \chi_{2} \mathrm{~d} y_{2} \mathrm{~d} y_{1}=\int_{E_{\mathrm{o}}-x_{1}} \int_{\mathbb{R}^{d}} \mathbf{1}\left\{x_{2}+y_{2}-y_{1} \in B_{t_{2}, r_{1}}\right\} \mathrm{d} y_{2} \mathrm{~d} y_{1}=\left|B_{t_{2}, r_{1}}\right|_{d}, \\
& \int_{\mathbb{R}^{d}} \int_{\mathbb{R}^{d}} \mathbf{1}\left\{x_{1}+y_{1} \in E_{\mathrm{o}}\right\} \mathbb{E} \kappa_{B}\left(x_{2}+y_{2}-x_{1}-y_{1} ; t_{1}, t_{2}, R\right) \mathrm{d} y_{2} \mathrm{~d} y_{1}=\mathbb{E}\left|B_{t_{1}, R}\right|_{d}\left|B_{t_{2}, R}\right|_{d}, \\
& \int_{\mathbb{R}^{d}} \int_{\mathbb{R}^{d}} \mathbf{1}\left\{x_{1}+y_{1} \in E_{\mathrm{o}}\right\} \mathbb{E} \tilde{\kappa}_{B}\left(x_{1}+y_{1}, x_{2}+y_{2} ; t_{1}, t_{2}, R\right) \mathrm{d} y_{2} \mathrm{~d} y_{1} \\
& =\int_{\mathbb{R}^{d}} \mathbf{1}\left\{x_{1}+y_{1} \in E_{\mathrm{o}}\right\} \mathbb{E}\left|\left(x_{1}+y_{1}+B_{t_{1}, R}\right) \cap F_{\mathrm{o}}^{c}\right|_{d}\left|B_{t_{2}, R}\right|_{d} \leq \mathbb{E}\left|\left(E_{\mathrm{o}} \oplus B_{t_{1}, R}\right) \cap F_{\mathrm{o}}^{c}\right|_{d}\left|B_{t_{2}, R}\right|_{d} .
\end{aligned}
$$


In the last two equations we used [20, Theorem 5.2.1]. Consequently, (2.12) and (2.11) yield

$$
\begin{aligned}
S_{2} \leq & \int_{C} \int_{C} \int_{0}^{\infty} \int_{0}^{\infty} f\left(t_{1}\right) f\left(t_{2}\right)\left[\left(\psi\left(t_{1}, r_{1}\right) \psi\left(t_{2}, r_{2}\right)+2 \psi\left(t_{2}, r_{2}\right)+2 \psi\left(t_{1}, r_{1}\right)\right)\right. \\
& \times\left(\gamma \mathbb{E}\left|B_{t_{1}, R}\right|_{d}\left|B_{t_{2}, R}\right|_{d}+\left|B_{t_{1}, r_{2}}\right|_{d}+\left|B_{t_{2}, r_{1}}\right|_{d}\right) \\
& +4\left(\nu\left(t_{1}\right) \nu\left(t_{2}\right)+\nu\left(t_{1}\right)+\nu\left(t_{2}\right)\right) \gamma \mathbb{E}\left|B_{t_{1}, R}\right|_{d}\left|B_{t_{2}, R}\right|_{d}+4 \gamma \mathbb{E}\left|\left(E_{\mathrm{o}} \oplus B_{t_{1}, R}\right) \cap F_{\mathrm{o}}^{c}\right|_{d}\left|B_{t_{2}, R}\right|_{d} \\
& +2\left(\left|B_{t_{1}, r_{2}}\right|_{d}+\left|B_{t_{2}, r_{1}}\right|_{d}\right)\left(\nu\left(t_{1}\right) \nu\left(t_{2}\right)+\sqrt{\nu\left(t_{1}\right) \nu\left(t_{2}\right)}\right)+\left|B_{t_{2}, r_{1}}\right|_{d} \nu\left(t_{1}\right)+\left|B_{t_{1}, r_{2}}\right|_{d} \nu\left(t_{2}\right) \\
& \left.+\left|B_{t_{1}, r_{2}}\right|_{d} \psi\left(t_{1}, r_{2}\right)+\left|B_{t_{2}, r_{1}}\right|_{d} \psi\left(t_{2}, r_{1}\right)\right] \mathrm{d} t_{2} \mathrm{~d} t_{1} \mathbb{G}\left(\mathrm{d} r_{2}\right) \mathbb{G}\left(\mathrm{d} r_{1}\right) .
\end{aligned}
$$

Using $\mathbb{E}\left|B_{t, R}\right|_{d} \leq \mathbb{E}\left|\left(E_{\mathrm{o}} \oplus B_{t, R}\right)\right|_{d} \leq c_{1}\left(1+t^{d}\right)$, cf. (3.11), the Cauchy-Schwarz inequality and assumption (3.1), it follows from the Lebesgue dominated convergence theorem that $S_{2} \rightarrow 0$ as $m \rightarrow \infty$.

Now we are dealing with the asymptotic normality of $\widehat{\mathbb{G}}_{n}(C)$.

Theorem 3.2. Assume that (3.1) is satisfied. Let $W_{n}=[-n, n)^{d}$. If $C \subset \mathbb{R}^{+}$is a Borel set, then

$$
\sqrt{\left|W_{n}\right|_{d}}\left(\widehat{\mathbb{G}}_{n}(C)-\mathbb{G}(C)\right) \underset{n \rightarrow \infty}{\stackrel{\mathrm{d}}{\longrightarrow}} N\left(0, \sigma_{\mathbb{G}}^{2}(C)\right),
$$

where

$$
\sigma_{\mathbb{G}}^{2}(C):=\frac{1}{\gamma^{2} \beta^{2}}\left[(1-\mathbb{G}(C)) \sigma^{2}(C)+\mathbb{G}(C) \sigma^{2}\left(\mathbb{R}^{+} \backslash C\right)-\mathbb{G}(C)(1-\mathbb{G}(C)) \sigma^{2}\left(\mathbb{R}^{+}\right)\right]
$$

and $\sigma^{2}(\cdot)$ is given by (2.14). If $0<\mathbb{G}(C)<1$, then $\sigma_{\mathbb{G}}^{2}(C)>0$.

Proof. Using (2.17) and Slutsky's theorem, the weak limit of $\sqrt{\left|W_{n}\right|_{d}}\left(\widehat{\mathbb{G}}_{n}(C)-\mathbb{G}(C)\right)$ coincides with the weak limit of

$$
Y_{n}:=\frac{1}{\gamma \beta \sqrt{\left|W_{n}\right|_{d}}}\left(\eta_{W_{n}}(C)-\eta_{W_{n}}\left(\mathbb{R}^{+}\right) \mathbb{G}(C)\right)
$$

Observing that

$$
\gamma \beta Y_{n}=\frac{1}{\sqrt{\left|W_{n}\right|_{d}}} \int_{W_{n}}\left(\mathbf{1}\left\{r_{B}(x, Z) \in C\right\}-\mathbb{G}(C)\right) f\left(d_{B}(x, Z)\right) h_{B}\left(d_{B}(x, Z), r_{B}(x, Z)\right)^{-1} \mathrm{~d} x,
$$

we can proceed along the same lines as in the proof of Theorem 3.1 and obtain

$$
\gamma \beta Y_{n} \underset{n \rightarrow \infty}{\stackrel{\mathrm{d}}{\longrightarrow}} N\left(0, \gamma^{2} \beta^{2} \sigma_{\mathbb{G}}^{2}(C)\right),
$$

provided we can identify the asymptotic variance $\sigma_{\mathbb{G}}^{2}(C)$ of $Y_{n}$. Theorem 2.4 implies that

$$
\begin{aligned}
\gamma^{2} \beta^{2} & \lim _{n \rightarrow \infty} \operatorname{Var} Y_{n} \\
& =\sigma^{2}(C)+\mathbb{G}(C)^{2} \sigma^{2}\left(\mathbb{R}^{+}\right)-2 \mathbb{G}(C) \lim _{n \rightarrow \infty} \frac{1}{\left|W_{n}\right|_{d}} \operatorname{Cov}\left(\eta_{W_{n}}(C), \eta_{W_{n}}\left(\mathbb{R}^{+}\right)\right) .
\end{aligned}
$$


Since $\eta_{W_{n}}(\cdot)$ is additive, we obtain from Theorem 2.4 that

$$
\begin{aligned}
2 \lim _{n \rightarrow \infty} & \frac{1}{\left|W_{n}\right|_{d}} \operatorname{Cov}\left(\eta_{W_{n}}(C), \eta_{W_{n}}\left(\mathbb{R}^{+}\right)\right)=2 \sigma^{2}(C)+2 \lim _{n \rightarrow \infty} \frac{1}{\left|W_{n}\right|_{d}} \operatorname{Cov}\left(\eta_{W_{n}}(C), \eta_{W_{n}}\left(\mathbb{R}^{+} \backslash C\right)\right) \\
= & 2 \sigma^{2}(C)+\lim _{n \rightarrow \infty} \frac{1}{\left|W_{n}\right|_{d}}\left(\operatorname{Var} \eta_{W_{n}}\left(\mathbb{R}^{+}\right)-\operatorname{Var} \eta_{W_{n}}(C)-\operatorname{Var} \eta_{W_{n}}\left(\mathbb{R}^{+} \backslash C\right)\right) \\
& =2 \sigma^{2}(C)+\sigma^{2}\left(\mathbb{R}^{+}\right)-\sigma^{2}(C)-\sigma^{2}\left(\mathbb{R}^{+} \backslash C\right)=\sigma^{2}(C)+\sigma^{2}\left(\mathbb{R}^{+}\right)-\sigma^{2}\left(\mathbb{R}^{+} \backslash C\right) .
\end{aligned}
$$

Inserting this result into (3.17) we obtain (3.16) upon some simplification.

To prove the last assertion, we define $\tilde{g}(t, s):=(\mathbf{1}\{s \in C\}-\mathbb{G}(C)) f(t) h_{B}(t, s)^{-1}$ and assume that $0<\mathbb{G}(C)<1$. For a convex body $W \subset \mathbb{R}^{d}$ we need to consider the variance of

$$
H_{W}:=\int_{W} \tilde{g}\left(d_{B}(x, Z), r_{B}(x, Z)\right) \mathrm{d} x
$$

As in the proof of the positivity assertion in Theorem 2.4 we obtain that

$$
\operatorname{Var} H_{W} \geq \gamma \int_{0}^{\infty} \int_{\mathbb{R}^{d}} \tilde{h}(y, r)^{2} \mathrm{~d} y \mathbb{G}(\mathrm{d} r),
$$

where

$$
\begin{aligned}
\tilde{h}(y, r):= & \mathbb{E} \int_{W} \mathbf{1}\left\{d_{B}(\mathrm{o}, B(y-x, r))<d_{B}(\mathrm{o}, Z)\right\} \tilde{g}\left(d_{B}(\mathrm{o}, B(y-x, r)), r\right) \mathrm{d} x \\
& -\mathbb{E} \int_{W} \mathbf{1}\left\{d_{B}(\mathrm{o}, B(y-x, r))<d_{B}(\mathrm{o}, Z)\right\} \tilde{g}\left(d_{B}(\mathrm{o}, Z), r_{B}(\mathrm{o}, Z)\right) \mathrm{d} x .
\end{aligned}
$$

By (1.4) and the definition of $\tilde{g}$ the second expectation on the above right-hand side vanishes for all $y \in W$ and $r \geq 0$. Therefore

$$
\tilde{h}(y, r)=\int_{W} \bar{F}_{B}\left(d_{B}(\mathrm{o}, B(y-x, r))\right) \tilde{g}\left(d_{B}(\mathrm{o}, B(y-x, r)), r\right) \mathrm{d} x .
$$

Again as in the proof of Theorem 2.4 we let $C^{\prime}:=\mathbb{R}^{+} \backslash C$ and obtain from Jensen's inequality and (3.18) that

$$
\begin{aligned}
\frac{\sqrt{\operatorname{Var} H_{W}}}{\sqrt{|W|_{d}}} \geq & \frac{c}{|W|_{d}} \int_{C^{\prime}} \int_{W} \int_{W} \bar{F}_{B}\left(d_{B}(\mathrm{o}, B(y-x, r))\right) f\left(d_{B}(\mathrm{o}, B(y-x, r))\right) \\
& \times\left(h_{B}\left(d_{B}(\mathrm{o}, B(y-x, r)), r\right)\right)^{-1} \mathrm{~d} x \mathrm{~d} y \mathbb{G}(\mathrm{d} r) \\
= & \frac{c}{|W|_{d}} \int_{C^{\prime}} \int_{\mathbb{R}^{d}}|W \cap(W-y)|_{d} \bar{F}_{B}\left(d_{B}(\mathrm{o}, B(y, r))\right) f\left(d_{B}(\mathrm{o}, B(y, r))\right) \\
& \times\left(h_{B}\left(d_{B}(\mathrm{o}, B(y, r)), r\right)\right)^{-1} \mathrm{~d} y \mathbb{G}(\mathrm{d} r),
\end{aligned}
$$

where $c>0$ is a constant not depending on $W$. Hence it is sufficient to show that

$$
\int_{C^{\prime}} \int_{\mathbb{R}^{d}} \bar{F}_{B}\left(d_{B}(\mathrm{o}, B(y, r))\right) f\left(d_{B}(\mathrm{o}, B(y, r))\right)\left(h_{B}\left(d_{B}(\mathrm{o}, B(y, r)), r\right)\right)^{-1} \mathrm{~d} y \mathbb{G}(\mathrm{d} r)>0 .
$$

By (2.12) the above integral equals $\mathbb{G}\left(C^{\prime}\right) \int_{\mathbb{R}^{d}} \bar{F}_{B}(t) f(t) \mathrm{d} t$, which is positive by (1.6). 
Remark 3.3. After some manipulation we get

$$
\gamma^{2} \beta^{2} \sigma_{\mathbb{G}^{2}}^{2}(C)=\gamma \int_{\mathbb{R}^{d}} \tilde{\tau}_{1}(C, u) \mathrm{d} u+\gamma^{2} \int_{\mathbb{R}^{d}} \tilde{\tau}_{2}(C, u) \mathrm{d} u
$$

where

$$
\begin{aligned}
\tilde{\tau}_{1}(C, u):=\int_{0}^{\infty} \int_{\mathbb{R}^{d}} \frac{f\left(d_{B}(\mathrm{o}, B(x, r))\right)}{h_{B}\left(d_{B}(\mathrm{o}, B(x, r)), r\right)} \frac{f\left(d_{B}(u, B(x, r))\right)}{h_{B}\left(d_{B}(u, B(x, r)), r\right)} \\
\quad \times \bar{F}_{B}^{(2)}\left(u ; d_{B}(\mathrm{o}, B(x, r)), d_{B}(u, B(x, r))\right)(\mathbf{1}\{r \in C\}-\mathbb{G}(C))^{2} \mathrm{~d} x \mathbb{G}(\mathrm{d} r)
\end{aligned}
$$

and

$$
\begin{aligned}
& \tilde{\tau}_{2}(C, u) \\
& :=\int_{0}^{\infty} \int_{0}^{\infty} \int_{\mathbb{R}^{d}} \int_{\mathbb{R}^{d}} \frac{f\left(d_{B}\left(x_{1}, B\left(\mathrm{o}, r_{1}\right)\right)\right)}{h_{B}\left(d_{B}\left(x_{2}, B\left(\mathrm{o}, r_{1}\right)\right), r_{1}\right)} \frac{f\left(d_{B}\left(x_{2}, B\left(\mathrm{o}, r_{2}\right)\right)\right)}{h_{B}\left(d_{B}\left(x_{2}, B\left(\mathrm{o}, r_{2}\right)\right), r_{2}\right)} \\
& \quad \times \mathbf{1}\left\{d_{B}\left(x_{2}, B\left(u, r_{2}\right)\right) \leq d_{B}\left(x_{1}, B\left(\mathrm{o}, r_{1}\right)\right)\right\} \mathbf{1}\left\{d_{B}\left(x_{1}, B\left(-u, r_{1}\right)\right) \leq d_{B}\left(x_{2}, B\left(\mathrm{o}, r_{2}\right)\right)\right\} \\
& \quad \times \bar{F}_{B}^{(2)}\left(u ; d_{B}\left(x_{1}, B\left(\mathrm{o}, r_{1}\right)\right), d_{B}\left(x_{2}, B\left(\mathrm{o}, r_{2}\right)\right)\right)\left(\mathbf{1}\left\{r_{1} \in C\right\}-\mathbb{G}(C)\right)\left(\mathbf{1}\left\{r_{2} \in C\right\}-\mathbb{G}(C)\right) \\
& \quad \times \mathrm{d} x_{1} \mathrm{~d} x_{2} \mathbb{G}\left(\mathrm{d} r_{1}\right) \mathbb{G}\left(\mathrm{d} r_{2}\right) .
\end{aligned}
$$

This relation can be also obtained directly by an analogue of the proof of Theorem 2.4

\section{The planar case}

We mentioned at the beginning that the estimator $\widehat{\mathbb{G}}$ which we discussed so far is based on the data $\left\{\left(d_{B}(x, Z), r_{B}(x, Z)\right): x \in W \backslash Z\right\}$ and therefore may require information from outside the window $W$. To overcome this problem, a common procedure in spatial statistics is the so-called minus sampling, which can be used, e.g., if the radius distribution $\mathbb{G}$ is concentrated on an interval $\left[0, r_{0}\right], 0<r_{0}<\infty$. We can avoid such a condition by assuming that the function $f$ is concentrated on an interval $[0, \varepsilon]$ with $\varepsilon>0$. If we then assume that $Z$ is observable in a window $W^{(\varepsilon)}$ which contains $W \oplus \varepsilon B$, then, for each $x \in W$, we have either $f\left(d_{B}(x, Z)\right)=0$ or $d_{B}(x, Z) \leq \varepsilon$, in which case the (almost surely unique) contact point $\left(x+d_{B}(x, Z) B\right) \cap Z$ lies in $W^{(\varepsilon)}$.

For practical applications, the planar case $d=2$ is particularly important. Also, then, the spherical case $B=B^{2}$ and the linear case $B=[0, u]$ (with a given direction $u$ ) play a major role. For simplicity, in the following considerations we concentrate on the window $W=[0,1]^{2}$ and we assume, as explained above, that $f$ is concentrated on $[0, \varepsilon], \varepsilon>0$, and that $Z$ is observed in $W^{(\varepsilon)}$. Let $\tilde{C}_{1}, \ldots, \tilde{C}_{k}$ be the (connected and relatively open) visible arcs in $\partial Z \cap W^{(\varepsilon)}$. We need not know whether some of these arcs belong to the same particle. By $C_{1} \subset \tilde{C}_{1}, \ldots, C_{k} \subset \tilde{C}_{k}$ we denote the corresponding "effective" arcs; these consist of the points $\left(x+d_{B}(x, Z) B\right) \cap Z \in \tilde{C}_{i}, x \in W \backslash Z$, for which $d_{B}(x, Z) \leq \varepsilon$. Let $r_{i}$ be the radius and $l_{i}$ the length of $C_{i}$, and let $A_{i}$ be the set of points $x \in W \backslash Z$ with $d_{B}(x, Z) \leq \varepsilon$ and which project onto $C_{i}, i=1, \ldots, k$, in the sense that $\left(x+d_{B}(x, Z) B\right) \cap Z$ consists of a unique point and this point lies in $C_{i}$, for $x \in A_{i}$. Then our estimator $\widehat{\mathbb{G}}$ is of the form

$$
\widehat{\mathbb{G}}=\frac{1}{\sum_{i=1}^{k} w_{i}} \sum_{i=1}^{k} w_{i} \delta_{r_{i}},
$$


where the weight $w_{i}$ is given by

$$
w_{i}=\int_{A_{i}} f\left(d_{B}(x, Z)\right) h_{B}\left(d_{B}(x, Z), r_{B}(x, Z)\right)^{-1} \mathrm{~d} x
$$

For $B=B^{2}$ we have $h_{B^{2}}(t, r)=2 \pi(t+r)$ (see Remark 2.3), hence if $f(t)=\varepsilon^{-1} \mathbf{1}\{t \leq \varepsilon\}$ then

$$
w_{i}=\frac{1}{2 \pi \varepsilon} \int_{A_{i} \cap\left(C_{i}+\varepsilon B^{2}\right)} \frac{1}{d_{B^{2}}\left(x, C_{i}\right)+r_{i}} \mathrm{~d} x .
$$

If we let $\varepsilon \rightarrow 0$, the weights converge to $w_{i}=l_{i} /\left(2 \pi r_{i}\right)$ if $r_{i}>0$ and to $w_{i}=1$ if $r_{i}=l_{i}=0$. Then the estimator becomes

$$
\widehat{\mathbb{G}}_{o}:=\left(\sum_{i=1}^{k} \frac{l_{i}}{r_{i}}\right)^{-1} \sum_{i=1}^{k} \frac{l_{i}}{r_{i}} \delta_{r_{i}}
$$

with $l_{i} / r_{i}$ interpreted as $2 \pi$ if $r_{i}=l_{i}=0$. Notice also that the outer sampling window $W^{(\varepsilon)}$ then shrinks to $W$, so that in the limit only information in $W$ is needed. The estimator $\widehat{\mathbb{G}}_{O}$ was discussed by Hall [3, Chapter 5.6] (more generally, he considered estimators of $\mathbb{E} A(R)$, for a given function $A$; $\widehat{\mathbb{G}}_{O}$ corresponds to the case $A=\mathbf{1}_{C}$ ).

For $B=[0, u]$ (with $u \in\left\{ \pm e_{1}, \pm e_{2}\right\}$ ), assuming (in the linear case) that $\mathbb{G}(\{0\})=0$ and hence $r_{i}>0$, and again choosing $f(t)=\varepsilon^{-1} \mathbf{1}\{t \leq \varepsilon\}$, we get $h_{B}(t, r)=2 r$ and

$$
w_{i}=\frac{1}{2 \varepsilon r_{i}} \int_{A_{i} \cap\left(C_{i}+\varepsilon[0,-u]\right)} \mathrm{d} x .
$$

This yields an estimator $\widehat{\mathbb{G}}_{l, u}$ in the limit $\varepsilon \rightarrow 0$, which is given by

$$
\widehat{\mathbb{G}}_{l, u}:=\left(\sum_{i=1}^{k} \frac{l_{i}(u)}{r_{i}}\right)^{-1} \sum_{i=1}^{k} \frac{l_{i}(u)}{r_{i}} \delta_{r_{i}} .
$$

Here, $l_{i}(u)$ is the length of the projection of the visible part of $C_{i}$ in direction $u$ (projected onto the line orthogonal to $u$ ). The estimator can be improved by combining $u=e_{1},-e_{1}, e_{2},-e_{2}$,

$$
\widehat{\mathbb{G}}_{l}:=\frac{1}{4}\left(\widehat{\mathbb{G}}_{l, e_{1}}+\widehat{\mathbb{G}}_{l,-e_{1}}+\widehat{\mathbb{G}}_{l, e_{2}}+\widehat{\mathbb{G}}_{l,-e_{2}}\right) \text {. }
$$

For applications, it would be natural to choose $\varepsilon=1$ which yields weights

$$
w_{i}=\frac{\left|A_{i}\right|_{2}}{2 r_{i}}, \quad i=1, \ldots, k,
$$

and gives the estimator

$$
\widehat{\mathbb{G}}=\left(\sum_{i=1}^{k} \frac{\left|A_{i}\right|_{2}}{r_{i}}\right)^{-1} \sum_{i=1}^{k} \frac{\left|A_{i}\right|_{2}}{r_{i}} \delta_{r_{i}} .
$$

Hence, in this case and with $u=e_{1}$, information in $[0,2] \times[0,1]$ would be required and the estimation is based on the areas of the regions $A_{i} \subset[0,1]^{2}$. Of course, the estimation can be again improved by combining the estimators for $u=e_{1},-e_{1}, e_{2},-e_{2}$ which are available if $Z$ is observed in $[-1,2]^{2}$.

If we do not have information from outside $W$, then we may use a minus sampling approach and replace $W$ by the eroded window $W_{\ominus \varepsilon}:=\{x \in W: x+\varepsilon B \subset W\}$, i.e. we consider the following estimator

$$
\widehat{\mathbb{G}}_{\ominus \varepsilon}(C):=\frac{\eta_{W_{\ominus \varepsilon}}(C)}{\eta_{W_{\ominus \varepsilon}}\left(\mathbb{R}^{+}\right)}
$$


Another possibility would be to use the naive approach which ignores edge effects. Then we have the uncorrected estimator

$$
\widehat{\mathbb{G}}_{\mathrm{u}}(C):=\frac{\eta_{W, \mathrm{u}}(C)}{\eta_{W, \mathrm{u}}\left(\mathbb{R}^{+}\right)},
$$

where

$$
\eta_{W, \mathrm{u}}(C):=\int_{W} 1\left\{r_{B}(x, Z \cap W) \in C\right\} \frac{f\left(d_{B}(x, Z \cap W)\right)}{h_{B}\left(d_{B}(x, Z \cap W), r_{B}(x, Z \cap W)\right)} \mathrm{d} x .
$$

If $B=[0, u]$, then it can happen that $d_{B}(x, Z \cap W)=\infty$. In that case we use our convention concerning $f \cdot h_{B}^{-1}$, i.e. the points $x$ satisfying $d_{B}(x, Z \cap W)=\infty$ do not contribute to $\eta_{W, \mathrm{u}}(C)$. Besides minus sampling there exist more sophisticated methods of edge correction in the statistics of spatial point processes. We adopt the idea of local minus sampling that was originally applied in [4] to the estimation of the nearest neighbour distance distribution function for stationary point processes (see also [5]). We use only points that are closer to $Z$ than to the boundary of the window $W$. This gives the Hanisch type estimator

$$
\widehat{\mathbb{G}}_{\mathrm{H}}(C):=\frac{\eta_{W, \mathrm{H}}(C)}{\eta_{W, \mathrm{H}}\left(\mathbb{R}^{+}\right)},
$$

where

$$
\eta_{W, \mathrm{H}}(C):=\int_{W} \mathbf{1}\left\{r_{B}(x, Z) \in C\right\} \mathbf{1}\left\{d_{B}(x, Z) \leq d_{B}(x, \partial W)\right\} \frac{f\left(d_{B}(x, Z)\right)}{h_{B}\left(d_{B}(x, Z), r_{B}(x, Z)\right)} \mathrm{d} x .
$$

Note that for $B=[0, u]$ the estimators $\widehat{\mathbb{G}}_{\mathrm{H}}$ and $\widehat{\mathbb{G}}_{\mathrm{u}}$ coincide.

In practical applications one has to replace in (1.5) the integration with respect to Lebesgue measure by an integration with respect to a discrete measure. This still gives a ratio-unbiased estimator of $\mathbb{G}$.

We compare the performance of the different estimators discussed above through computer simulations. We simulate a stationary planar Boolean model with spherical grains, given by (1.1). The observation window $W$ is the unit square $[0,1]^{2}$. The distribution $\mathbb{G}$ is assumed to be uniform on $(0.05,0.1)$. We approximate the integrals over $W$ by Riemannian sums over a rectangular grid of points $L_{h} \cap W$, where

$$
L_{h}:=\{((k-1 / 2) h,(l-1 / 2) h): k, l \in \mathbb{N}\} .
$$

For our purposes, we choose $h=1 / 300$.

We take $f(t)=\varepsilon^{-1} \mathbf{1}\{t \leq \varepsilon\}$ for different choices of $\varepsilon$ and compare the estimator $\widehat{\mathbb{G}}$, given by (1.8), with the estimators $\widehat{\mathbb{G}}_{o}$ (for spherical $B$ ) and $\widehat{\mathbb{G}}_{l}$ (for linear $B$ ) corresponding to the limiting case $\varepsilon \rightarrow 0$. The estimators $\widehat{\mathbb{G}}_{\ominus \varepsilon}, \widehat{\mathbb{G}}_{\mathrm{u}}$ and $\widehat{\mathbb{G}}_{\mathrm{H}}$ are also evaluated. For linear $B=[0, u]$ we always combine the corresponding estimators for $u=e_{1},-e_{1}, e_{2},-e_{2}$, this leads to a noticeable improvement.

The radius distribution $\mathbb{G}$ is uniquely determined by the distribution function $G(t)=\mathbb{G}([0, t]), t \geq 0$. We measure the quality of the estimators by the Kolmogorov-Smirnov distance

$$
d_{\mathrm{KS}}(\widehat{G}, G):=\sup _{s \geq 0}|\widehat{G}(s)-G(s)|
$$

and the Cramér-von Mises distance

$$
d_{\mathrm{CvM}}(\widehat{G}, G):=\int_{0.05}^{0.1}(\widehat{G}(s)-G(s))^{2} \frac{\mathrm{d} s}{0.05} .
$$

We have generated 100 independent realizations of the Boolean model $Z$ with chosen intensity $\gamma$. For each realization we have determined several estimators under study. The sample means of corresponding Kolmogorov-Smirnov and Cramér-von Mises distances over 100 simulations are demonstrated in Table 1 for $\gamma=25$ and in Table 2 for $\gamma=100$. The results show that smaller values of $\varepsilon$ are more 


\begin{tabular}{|c|cc|cc|}
\hline & \multicolumn{2}{|c|}{$d_{\mathrm{KS}}$} & \multicolumn{2}{c|}{$1000 \cdot d_{\mathrm{CvM}}$} \\
estimator & spherical $B$ & linear $B$ & spherical $B$ & linear $B$ \\
\hline$\widehat{\mathbb{G}}, \varepsilon=1$ & 0.178 & 0.147 & 7.921 & 5.139 \\
$\mathbb{G}, \varepsilon=0.05$ & 0.172 & 0.170 & 7.317 & 7.101 \\
$\widehat{\mathbb{G}}, \varepsilon=0.01$ & 0.172 & 0.172 & 7.295 & 7.292 \\
\hline$\widehat{\mathbb{G}}_{o}$ or $\widehat{\mathbb{G}}_{l}$ & 0.171 & 0.172 & 7.243 & 7.257 \\
\hline$\widehat{\mathbb{G}}_{\ominus \varepsilon}, \varepsilon=0.05$ & 0.191 & 0.177 & 9.243 & 7.753 \\
$\widehat{\mathbb{G}}_{\ominus \varepsilon}, \varepsilon=0.01$ & 0.176 & 0.173 & 7.674 & 7.435 \\
\hline$\widehat{\mathbb{G}}_{\mathrm{u}}, \varepsilon=1$ & 0.182 & 0.179 & 8.389 & 7.890 \\
$\widehat{\mathbb{G}}_{\mathrm{u}}, \varepsilon=0.05$ & 0.173 & 0.169 & 7.480 & 7.553 \\
$\widehat{\mathbb{G}}_{\mathrm{u}}, \varepsilon=0.01$ & 0.173 & 0.168 & 7.322 & 7.472 \\
\hline$\widehat{\mathbb{G}}_{\mathrm{H}}, \varepsilon=1$ & 0.187 & 0.179 & 9.003 & 7.890 \\
$\widehat{\mathbb{G}}_{\mathrm{H}}, \varepsilon=0.05$ & 0.179 & 0.169 & 8.023 & 7.553 \\
$\widehat{\mathbb{G}}_{\mathrm{H}}, \varepsilon=0.01$ & 0.174 & 0.168 & 7.462 & 7.472 \\
\hline
\end{tabular}

Table 1: Sample means of distances between distribution functions computed from 100 realizations of a Boolean model with intensity $\gamma=25$ and uniform radius distribution on $(0.05,0.1)$.

preferable. The limiting estimators $\widehat{\mathbb{G}}_{o}$ and $\widehat{\mathbb{G}}_{l}$ produced the smallest error. They are outperformed only in the case of smaller intensity and linear $B$ where our estimator, given by (1.8), with larger $\varepsilon$, gives better results. However, this estimator uses also information from outside $W$. Simulation studies for exponentially distributed radii (not presented) show very similar results. A change of resolution $h$ has only a minor influence on the quality of the estimators. For intensity $\gamma \gg 100$ the deviation from the radius distribution increases which is intuitively clear because many balls are covered so that their radii are not available for the estimators.

\section{References}

[1] Ballani, F. On second-order characteristics of germ-grain models with convex grains, Mathematika 53 (2006), 255-285.

[2] Billingsley, P. Convergence of Probability Measures, 2nd edition, John Wiley \& Sons, New York, 1999.

[3] Hall, P. Introduction to the Theory of Coverage Processes, John Wiley \& Sons, New York, 1988.

[4] Hanisch, K.-H. Some remarks on estimators of the distribution function of nearest neighbour distance in stationary spatial point patterns, Statistics 15 (1984), 409-412.

[5] Hansen, M. B., Baddeley, A. J., Gill, R. D. First contact distributions for spatial patterns: regularity and estimation, Adv. Appl. Prob. (SGSA) 31 (1999), 15-33.

[6] Heinrich, L. Asymptotic methods in statistics of random point processes, In: Spodarev, E. (ed) Stochastic Geometry, Spatial Statistics and Random Fields, pp. 115-150, Lecture Notes in Mathematics 2068. Springer, Berlin, 2013.

[7] Heinrich, L., Molchanov, I. S. Central limit theorem for a class of random measures associated with germ-grain models, Adv. Appl. Prob. (SGSA) 31 (1999), 283-314. 


\begin{tabular}{|c|cc|cc|}
\hline & \multicolumn{2}{|c|}{$d_{\mathrm{KS}}$} & \multicolumn{2}{c|}{$1000 \cdot d_{\mathrm{CvM}}$} \\
estimator & spherical $B$ & linear $B$ & spherical $B$ & linear $B$ \\
\hline$\widehat{\mathbb{G}}, \varepsilon=1$ & 0.147 & 0.134 & 5.506 & 4.406 \\
$\mathbb{\mathbb { G }}, \varepsilon=0.05$ & 0.145 & 0.131 & 5.294 & 4.238 \\
$\widehat{\mathbb{G}}, \varepsilon=0.01$ & 0.132 & 0.128 & 4.276 & 4.008 \\
\hline$\widehat{\mathbb{G}}_{o}$ or $\widehat{\mathbb{G}}_{l}$ & 0.127 & 0.127 & 3.919 & 3.928 \\
\hline$\widehat{\mathbb{G}}_{\ominus \varepsilon}, \varepsilon=0.05$ & 0.158 & 0.135 & 6.162 & 4.460 \\
$\widehat{\mathbb{G}}_{\ominus \varepsilon}, \varepsilon=0.01$ & 0.134 & 0.129 & 4.359 & 4.029 \\
\hline$\widehat{\mathbb{G}}_{\mathrm{u}}, \varepsilon=1$ & 0.150 & 0.140 & 5.710 & 4.838 \\
$\widehat{\mathbb{G}}_{\mathrm{u}}, \varepsilon=0.05$ & 0.147 & 0.137 & 5.431 & 4.807 \\
$\widehat{\mathbb{G}}_{\mathrm{u}}, \varepsilon=0.01$ & 0.133 & 0.129 & 4.299 & 4.208 \\
\hline$\widehat{\mathbb{G}}_{\mathrm{H}}, \varepsilon=1$ & 0.150 & 0.140 & 5.602 & 4.838 \\
$\widehat{\mathbb{G}}_{\mathrm{H}}, \varepsilon=0.05$ & 0.148 & 0.137 & 5.438 & 4.807 \\
$\widehat{\mathbb{G}}_{\mathrm{H}}, \varepsilon=0.01$ & 0.133 & 0.129 & 4.323 & 4.208 \\
\hline
\end{tabular}

Table 2: Sample means of distances between distribution functions computed from 100 realizations of a Boolean model with intensity $\gamma=100$ and uniform radius distribution on $(0.05,0.1)$.

[8] Heinrich, L., Werner, W. Kernel estimation of the diameter distribution in Boolean models with spherical grains, J. Nonparametr. Statist. 12 (2000), 147-176.

[9] Hug, D., Last, G. On support measures in Minkowski spaces and contact distributions in stochastic geometry, Ann. Probab. 28 (2000), 796-850.

[10] Hug, D., Last, G., Weil, W. Generalized contact distributions of inhomogeneous Boolean models, Adv. Appl. Prob. (SGSA) 34 (2002), 21-47.

[11] Kallenberg, O. Foundations of Modern Probability, 2nd edition, Springer-Verlag, New York, 2002.

[12] Kiderlen, M., Weil, W. Measure-valued valuations and mixed curvature measures of convex bodies, Geom. Dedicata 76 (1999), 291-329.

[13] Last, G., Penrose, M. D. Fock space representation, chaos expansion and covariance inequalities for general Poisson processes, Prob. Theory Rel. Fields 150 (2011), 663-690.

[14] Molchanov, I. S. Estimation of the size distribution of spherical grains in the Boolean model, Biometrical J. 32 (1990), 877-886.

[15] Molchanov, I. S. Statistics of the Boolean Model for Practitioners and Mathematicians, Wiley, Chichester, 1997.

[16] Molchanov, I. S., Stoyan, D. Asymptotic properties of estimators for parameters of the Boolean model, Adv. Appl. Prob. 27 (1994), 63-86.

[17] Rosén, B. A note on asymptotic normality of sums of higher-dimensionally indexed random variables, Ark. Mat. 8 (1969), 33-43.

[18] Schneider, R. Convex Bodies: The Brunn-Minkowski Theory, Cambridge University Press, Cambridge, 1993.

[19] Stoyan, D., Kendall, W. S., Mecke, J. Stochastic Geometry and its Applications, 2nd edition, Wiley, Chichester, 1995. 
[20] Schneider, R., Weil, W. Stochastic and Integral Geometry, Springer-Verlag, Berlin, 2008.

Authors' addresses:

Daniel Hug, Karlsruhe Institute of Technology (KIT), Department of Mathematics, e-mail: daniel.hug@kit.edu

Günter Last, Karlsruhe Institute of Technology (KIT), Department of Mathematics, e-mail: guenter.last@kit.edu

Zbyněk Pawlas, Department of Probability and Mathematical Statistics, Faculty of Mathematics and Physics, Charles University, Sokolovská 83, 18675 Praha 8, Czech Republic,

e-mail: pawlas@karlin.mff.cuni.cz

Wolfgang Weil, Karlsruhe Institute of Technology (KIT), Department of Mathematics, e-mail: wolfgang.weil@kit.edu 